1 Title: Extensive genetic diversity of bat-borne polyomaviruses reveals inter-family

2 host-switching events

3 Short title: Host-switching of polyomaviruses in bats

4 Author names and affiliations:

5 Zhizhou Tan ${ }^{1 \uparrow}$, Gabriel Gonzalez ${ }^{2 \uparrow}$, Jinliang Sheng ${ }^{3 \uparrow, ~ J i a n m i n ~} \mathrm{Wu}^{4}$, Fuqiang Zhang ${ }^{5}$, Lin $\mathrm{Xu}^{1}$,

6 Peisheng Zhang ${ }^{1,3}$, Aiwei Zhu'1, Yonggang Qu ${ }^{3}$, Changchun Tu${ }^{1,6^{*}}$, Michael J. Carr ${ }^{7 *}$, Biao He $\mathrm{He}^{1,6^{*}}$

$8 \quad{ }^{1}$ Key Laboratory of Jilin Province for Zoonosis Prevention and Control, Institute of Military

9 Veterinary Medicine, Academy of Military Medical Sciences, Changchun, Jilin Province, China.

$10{ }^{2}$ National Advanced Computing Collaboratory, National Center for High Technology, San Jose,

11 Costa Rica.

$12{ }^{3}$ College of Animal Science and Technology, Shihezi University, Shihezi, Xinjiang Uyghur

13 Autonomous Region, China.

$14{ }^{4}$ Guangxi Key Laboratory of Veterinary Biotechnology, Guangxi Veterinary Research Institute,

15 Nanning, Guangxi Province, China.

$16{ }^{5}$ Center for Disease Control and Prevention of Southern Theater Command, Kunming, Yunnan

17 Province, China.

$18{ }^{6}$ Jiangsu Co-innovation Center for Prevention and Control of Important Animal Infectious

19 Diseases and Zoonosis, Yangzhou, Jiangsu Province, China.

$20{ }^{7}$ National Virus Reference Laboratory, School of Medicine, University College Dublin, Dublin 4, 21 Ireland. 


\section{$22{ }^{*}$ Corresponding authors:}

23 heb-001001@163.com (Biao He);

24 michael.carr@ucd.ie (Michael J. Carr);

25 changchun_tu@hotmail.com (Changchun Tu).

26

27 TThese authors contributed equally to this work. 


\section{Abstract}

30 Polyomaviruses (PyVs) are small, double-stranded DNA tumor viruses carried by diverse

31 vertebrates. PyVs have previously been considered highly host restricted in mammalian hosts,

32 with host-switching events thought rare or nonexistent. Prior investigations have revealed

33 short-range host-switching events of PyVs in two different African bat species within the

34 horseshoe bat genus Rhinolophus. Herein, we have conducted a systematic investigation of PyVs

35 in 1,083 archived bat samples collected from five provinces across China, and identified 192

36 PyVs from 186 bats from 15 host species within 6 families (Rhinolophidae, Vespertilionidae,

37 Hipposideridae, Emballonuridae, Miniopteridae and Pteropodidae) representing 28

newly-described PyVs, indicative of extensive genetic diversity of bat PyVs. Surprisingly, two

PyVs were identified in multiple bat species from different families, and another PyV clustered

phylogenetically with PyVs carried by bats from a different host family, indicative of three

44 explained by host-switching events. PyVs identified from geographically separated Chinese

45 horseshoe bat species in the present study showed close genetic identities, and clustered with

46 each other and with PyVs from African horseshoe bats, allowing assessment of the effects of

47 positive selection in VP1 within the horseshoe bat family Rhinolophidae. Correlation analysis

48 indicated that co-evolution with their hosts contributed much more to evolutionary divergence of

49 PyV than geographic distance. In conclusion, our findings provide the first evidence of 
50 inter-family host-switching events of PyV in mammals and challenge the prevailing evolutionary

51 paradigm for strict host restriction of mammalian PyVs.

54 Since the discovery of murine polyomavirus in the 1950s, polyomaviruses (PyVs) have been

55 considered both genetically stable and highly host-restricted in their mammalian hosts. In this

56 study, we have identified multiple cases of host-switching events of PyVs by large scale

57 surveillance in diverse bat species collected in China. These host-switching events occurred

58 between bat families living in the same colony, indicating that a large population with frequent

59 contacts between different bat species may represent an ecological niche facilitating PyV

60 host-switching. The cases studied involved members of bats from several families, including

61 horseshoe bats, which were previously found to harbor a number of highly virulent viruses to

62 both humans and domestic animals. Our findings have provided evidence that even highly

63 host-specific DNA viruses can transmit between bats of different species and indicate an

64 increased propensity for spillover events involving horseshoe bats. We propose an evolutionary

65 scheme for bat-borne PyVs in which intra-host divergence and host-switching has generated the

66 diverse PyVs in present day bats. This scheme provides a useful model to study the evolution of

67 PyVs in other hosts and, potentially, the modeling of bat zoonoses and the transmission of other

68 DNA viruses in other mammals, including humans. 


\section{Introduction}

71 Bats (order Chiroptera) account for more than $20 \%$ of mammalian species with $>1,100$ known

72 species in $>200$ genera worldwide [1]. These diverse mammals harbor numerous highly virulent

73 viral pathogens, including rabies virus [2], Marburg virus [3], Ebola virus [4], Nipah virus [5],

74 severe acute respiratory syndrome (SARS)-related coronavirus [6-8] and the newly identified

75 swine acute diarrhea syndrome coronavirus [9]. Spillover of viruses from bats to humans or

76 domestic animals can give rise to outbreaks of emerging infectious diseases [10].

77 Polyomaviruses (PyVs) are non-enveloped icosahedral DNA viruses containing a circular and

78 highly stable double-stranded DNA genome $[11,12]$. PyVs can induce neoplastic transformation

79 in cell culture [12], and are associated with malignancy in humans [13, 14] and raccoons [15].

80 Currently, 98 species carried by various mammals, birds and fish are recognized within the

81 family Polyomaviridae by the International Committee on Taxonomy of Viruses (ICTV) and

82 classified into four separate genera (Alpha-, Beta-, Gamma- and Deltapolyomavirus) with nine

83 floating species [16]. Recent identification of diverse PyV-like sequences in different fish species

84 [17] and invertebrates (including arachnids and insects) has revealed a deep co-evolutionary

85 history of PyVs with their metazoan hosts [17]. PyVs are highly host-specific in different

86 mammalian species, and long-range host jumps leading to productive infection and transmission

87 within mammalian genera are considered rare or nonexistent $[17,18]$. Recently, an intra-host

88 divergence model with contributions from viral lineage duplication, recombination and,

89 potentially, host-switching at evolutionary timescales has been proposed to explain the extant

90 diversity based on the available genetic data [17]. 
91 Currently, 15 alphapolyomaviruses and 10 betapolyomaviruses have been formally recognized

92 in insectivorous and frugivorous bat species within the Americas, Africa, Southeast Asia

93 (Indonesia) and the Pacific (New Zealand) [19-26]. These studies identified significant diversity

94 and high positive rates (10-20\%) using PCR-based approaches [27]. PyVs with complete or

95 partial genomes has also been identified by metagenomic analyses in bats collected in China and

96 recently from Saudi Arabia [28-30]. Investigation of PyVs in African insectivorous and

97 frugivorous bats $[25,26]$ has revealed evidence for short-range host-switching events of PyVs in

98 horseshoe bats (family Rhinolophidae, genus Rhinolophus) following identification of nearly

99 identical viruses (99.9\% at the genomic level) in Rh. blasii and Rh. simulator [26].

100 In the present study, we have characterized the diversity and genetic relationships of novel

101 PyVs from a large number of insectivorous and frugivorous bat samples collected in diverse

102 ecological environments in China, resulting in the identification of inter-family host-switching

103 events in these bat-borne PyVs. The findings challenge the prevailing paradigm of intra-host PyV

104 diversification in their mammalian hosts and extend the knowledge of PyV genetic diversity,

105 evolution, host restriction and host-switching.

106

107 Results

108 Sampling information and detection of polyomaviruses.

109 In 2015-2016, a total of 1,083 bats belonging to 20 species in 6 families (Rhinolophidae,

110 Vespertilionidae, Hipposideridae, Emballonuridae, Miniopteridae and Pteropodidae) were

111 collected from 29 different colonies (colonies 1-29 in Fig 1 and S1 Table) from five provinces in 
124 sequencing. A total of $1.76 \times 10^{7}$ sequence reads with an average length of 125 nucleotides (nt)

125 were obtained, of which 694 PyV-related reads with average length of 167 nt were identified

126 from 38,468 virus-related sequences. Preliminary analyses of these PyV sequences indicated a

127 high level of diversity with previously unrecognized members from both Alpha- and

\section{Betapolyomavirus genera.}

129 To determine the prevalence of PyVs in bats collected not only from Yunnan but also from 
133 positive samples from 15 bat species within 6 families. These PyV positive bats were from 21 of

13429 bat colonies in the survey area. As shown in Fig 1 and Table 1, all five investigated provinces

135 had PyV positive bats with detection rates of $9.6 \%(20 / 208)$ in Yunnan, 11.9\% (59/495) in

136 Guangxi, 10.1\% (16/158) in Xinjiang, 42.5\% (74/174) in Fujian and 35.4\% (17/48) in Zhejiang.

137 With respect to the bat families (Fig 1 and Table 1), 103 from 6 species of Rhinolophidae, 60

138 from 4 species of Vespertilionidae, 7 from 2 species of Hipposideridae, and 11, 2 and 3

139 respectively from 1 species each of Emballonuridae, Miniopteridae and Pteropodidae were PyV

140 positive. At the species level, Rh. affinis, in the Rhinolophidae, widely distributed in southern

141 China, yielded the highest number of positive bats $(\mathrm{n}=64)$, while Myotis horsfieldii in the

142 Vespertilionidae collected in Fujian, showed the highest positivity rate: $66.6 \%(24 / 36)$. 
bioRxiv preprint doi: https://doi.org/10.1101/627158; this version posted May 3, 2019. The copyright holder for this preprint (which was not certified by peer review) is the author/funder, who has granted bioRxiv a license to display the preprint in perpetuity. It is made available under aCC-BY 4.0 International license.

Table 1. Sampling of bats and positive rate (\%) of PyVs in Chinese provinces

\begin{tabular}{|c|c|c|c|c|}
\hline Bat family and total positive rate & Bat species and total positive rate & $\begin{array}{l}\text { Positive rate in } \\
\text { colonies }\end{array}$ & $\begin{array}{l}\text { Colony code } \\
\text { (Province and Prefecture) }^{\mathrm{a}}\end{array}$ & $\begin{array}{l}\begin{array}{l}\text { Number } \\
\text { of PyVs }\end{array} \\
\end{array}$ \\
\hline \multirow[t]{16}{*}{ Rhinolophidae, $24.9(103 / 413)$} & \multirow[t]{5}{*}{ Rhinolophus affinis, $34.0(64 / 188)$} & $15.0(9 / 60)$ & 1 (Yunnan Wenshan) & 4 \\
\hline & & $31.8(7 / 22)$ & 16 (Guangxi Fangchenggang) & 3 \\
\hline & & $44.4(12 / 27)$ & 17 (Guangxi Fangchenggang) & 4 \\
\hline & & $41.3(24 / 58)$ & 23 (Fujian Nanping) & 1 \\
\hline & & $57.1(12 / 21$ & 24 (Fujian Sanming) & 1 \\
\hline & Rhinolophus sp. CN 2016, $11.1(4 / 36)$ & $11.1(4 / 36)$ & 2 (Yunnan Baoshan) & 2 \\
\hline & \multirow[t]{2}{*}{ Rhinolophus thomasi, $8.6(3 / 35)$} & $7.1(2 / 28)$ & 4 (Yunnan Dehong) & 2 \\
\hline & & $14.2(1 / 7)$ & 20 (Guangxi Baise) & 1 \\
\hline & \multirow{3}{*}{ Rhinolophus pearsonii, $1.8(1 / 55)$} & $3.1(1 / 32)$ & 4 (Yunnan Dehong) & 2 \\
\hline & & $0(0 / 20)$ & 5 (Guangxi Hechi) & - \\
\hline & & $0(0 / 3)$ & 20 (Guangxi Baise) & - \\
\hline & Rhinolophus luctus, $0(0 / 1)$ & $0(0 / 1)$ & 4 (Yunnan Dehong) & \\
\hline & \multirow[t]{2}{*}{ Rhinolophus sinicus, $28.0(14 / 50)$} & $28.6(14 / 49)$ & 21 (Fujian Longyan) & 6 \\
\hline & & $0(0 / 1)$ & 22 (Fujian Nanping) & - \\
\hline & \multirow{2}{*}{ Rhinolophus ferrumequinum, 35.4 (17/48) } & $20.0(2 / 10)$ & 25 (Zhejiang Zhoushan) & 1 \\
\hline & & $39.5(15 / 38)$ & 26 (Zhejiang Zhoushan) & 4 \\
\hline \multirow[t]{9}{*}{ Vespertilionidae, $16.0(60 / 376)$} & \multirow[t]{4}{*}{ Scotophilus kuhlii, 6.3 (8/127) } & $3.2(1 / 31)$ & 8 (Guangxi Beihai) & 1 \\
\hline & & $10.0(4 / 40)$ & 9 (Guangxi Beihai) & 2 \\
\hline & & $8.0(1 / 13)$ & 10 (Guangxi Beihai) & 1 \\
\hline & & $4.7(2 / 43)$ & 18 (Guangxi Qinzhou) & 1 \\
\hline & Myotis davidii, $21.8(12 / 55)$ & $21.8(12 / 55)$ & 11 (Guangxi Guilin) & 4 \\
\hline & Myotis horsfieldii, 66.7 (24/36) & $66.7(24 / 36)$ & 24 (Fujian Sanming) & 3 \\
\hline & \multirow[t]{3}{*}{ Pipistrellus pipistrellus, $10.1(16 / 158)$} & $2.6(2 / 76)$ & 27 (Xinjiang Ili) & 1 \\
\hline & & $25.5(12 / 47)$ & 28 (Xinjiang Ili) & 3 \\
\hline & & $5.7(2 / 35)$ & 28 (Xinjiang Shihezi) & 1 \\
\hline \multirow{19}{*}{ Hipposideridae, $3.3(7 / 210)$} & \multirow{2}{*}{ Aselliscus stoliczkanus, $2.4(1 / 42)$} & $2.6(1 / 38)$ & 2 (Yunnan Baoshan) & 1 \\
\hline & & $0(0 / 4)$ & 19 (Guangxi Baise) & - \\
\hline & \multirow[t]{5}{*}{ Hipposideros armiger, $0(0 / 48)$} & $0(0 / 3)$ & 2 (Yunnan Baoshan) & - \\
\hline & & $0(0 / 21)$ & 15 (Guangxi Chongzuo) & - \\
\hline & & $0(0 / 9)$ & 19 (Guangxi Baise) & - \\
\hline & & $0(0 / 6)$ & 20 (Guangxi Baise) & - \\
\hline & & $0(0 / 9)$ & 22 (Fujian Nanping) & - \\
\hline & \multirow{6}{*}{ Hipposideros larvatus, $8.9 \%$ (6/68) } & $0(0 / 10)$ & 6 (Guangxi Nanning) & - \\
\hline & & $0(0 / 20)$ & 7 (Guangxi Nanning) & - \\
\hline & & $21.1(4 / 19)$ & 16 (Guangxi Fangchenggang) & 2 \\
\hline & & $20.0(2 / 10)$ & 17 (Guangxi Fangchenggang) & 1 \\
\hline & & $0(0 / 5)$ & 19 (Guangxi Baise) & - \\
\hline & & $0(0 / 4)$ & 20 (Guangxi Baise) & - \\
\hline & \multirow[t]{5}{*}{ Hipposideros pomona, $0(0 / 44)$} & $0(0 / 18)$ & 12 (Guangxi Liuzhou) & - \\
\hline & & $0(0 / 20)$ & 13 (Guangxi Laibin) & - \\
\hline & & $0(0 / 2)$ & 17 Guangxi Fangchenggang) & - \\
\hline & & $0(0 / 3)$ & 19 (Guangxi Baise) & - \\
\hline & & $0(0 / 1)$ & 20 (Guangxi Baise) & - \\
\hline & Hipposideros turpis, $0(0 / 8)$ & $0(0 / 8)$ & 19 (Guangxi Baise) & - \\
\hline Emballonuridae, 31.4 (11/35) & Taphozous melanopogon, 31.4 (11/35) & $31.4(11 / 35)$ & 15 (Guangxi Chongzuo) & 3 \\
\hline \multirow[t]{3}{*}{ Miniopteridae, $5.0(2 / 40)$} & \multirow{3}{*}{$\begin{array}{l}\text { Miniopterus australis, } 0(0 / 1) \\
\text { Miniopterus schreibersii, } 5.1 \text { (2/39) }\end{array}$} & $0(0 / 1)$ & 17 Guangxi Fangchenggang) & - \\
\hline & & $0(0 / 1)$ & 4 (Yunnan Dehong) & - \\
\hline & & $5.3(2 / 38)$ & 14 (Guangxi Chongzuo) & 2 \\
\hline Pteropodidae, 33.3 (3/9) & Rousettus leschenaultii, 33.3 (3/9) & $33.3(3 / 9)$ & 3 (Yunnan Baoshan) & 3 \\
\hline Total, $17.2(186 / 1,083)$ & - & - & - & - \\
\hline
\end{tabular}

a Number used to encode bat colonies and location as shown in Fig 1, with detailed information 146 provided in S1 Table.

${ }^{\mathrm{b}}$ Number of PyVs identified based on the partial VP1 sequences as shown in Fig 2 and S2 Table. 


\section{Extensive genetic diversity of polyomaviruses among bats.}

150 VP1 genes of PyV were amplified using broad-spectrum primers from 186 positive samples and

151 then cloned into vectors. Four clones derived from each amplicon were sequenced to detect

152 potential co-infection of multiple PyVs in each positive sample. As a result, 180 of 186 bats were

153 confirmed to harbor one PyV, while the remaining 6 had a co-infection with two distinct PyVs

154 (detailed in S1 Table). A total of 192 non-redundant partial VP1 sequences were obtained and

155 phylogenetically compared with VP1 sequences of known PyVs. Results showed that the 192

156 VP1 sequences had extensive diversity and could be classified into 44 clusters within the Alpha-

157 and Betapolyomavirus genera (Fig 2 and S1 Fig), with 46.6-90.1\% nt identities between clusters

158 and 95.1-100\% within clusters. Of these 44 PyVs clusters, 42 showed exclusive host specificity

159 within a single bat species while, surprisingly, the remaining 2 clusters included viruses detected

in two bat species within different families (Rh. affinis and Hipposideros larvatus; Rh. affinis and

Myotis horsfieldii, respectively). This finding provided preliminary evidence of inter-family

transmission of PyVs (Fig 2 and S1 Fig). The majority $(n=191)$ of these partial VP1 shared less

than $90 \%$ nt identity with the closest known PyVs. Interestingly, one VP1 sequence (marked by

cluster 10 in S1 Fig), named here as Pipistrellus pipistrellus polyomavirus XJPp02 (strain

PyV10-YXAC25) shared 98\% nt identity with Rattus norvegicus polyomavirus 1, previously

identified in brown rats in Germany [35].

To further characterize the genetic diversity of the PyVs in these bats, inverse PCR based on 
170

171

172

173

174

175

176

177

178

179

180

181

182

183

184

185

186

187

188

189

190

novel bat-borne PyV were 4,869-5,529 bp with $39.49-44.69 \% \mathrm{G}+\mathrm{C}$ content. All PyV genomes displayed an archetypal genomic organization, including an early region encoding two nonstructural proteins [large tumor antigen (LTAg), and short tumor antigen (STAg)], and a late region encoding three capsid proteins (VP1, VP2 and VP3) on opposite strands. Early and late regions were separated by a non-coding control region harboring regulatory elements. For all samples from which PyVs genomes were obtained, a $\sim 1,800$ bp fragment of the $c y t b$ gene was amplified to corroborate the bat host species (Table 2 and S2 Fig).

The PyV strains sharing $\geq 97 \%$ nt identity within the LTAg coding sequence were defined as the same PyV, while those sharing $\leq 90 \%$ identities were defined as different PyV [36]. Consequently, as shown in Table 2 and Fig 3, the $42 \mathrm{PyV}$ strains were identified as 28 novel bat-borne PyVs based on genomic identities. Of these novel bat-borne PyVs, 26 were identified in a single bat species, indicative of a high degree of host specificity. They included one PyV identified in a cryptic bat species (provisionally named Rhinolophus $s p$. CN2016) the permanent identification of which awaits formal binomial classification of the host species as the cytb gene showed only $90 \%$ nt identity with that of known bat species [37]. The PyVs named in the present study were based on the binomial names of their host bat species and the chronological order of discovery, as per ICTV guidelines. For example, Aselliscus stoliczkanus polyomavirus 1 was the first PyV discovered in Aselliscus stoliczkanus bats, and Rhinolophus affinis polyomavirus 2 was the second PyV discovered in Rh. affinis bats. Interestingly, two remaining PyVs were identified in more than one bat species. In detail, a PyV, herein provisionally named as Rhinolophus affinis-Hipposideros larvatus polyomavirus (Ra-Hl PyV), was identified in both $R h$. affinis and $H$. 
191 larvatus bats. Similarly, another PyV named as Rhinolophus affinis-Myotis horsfieldii

192 polyomavirus (Ra-Mh PyV) was identified in Rh. affinis and My. horsfieldii bats. For clarity and

193 according to the naming tradition of PyV, the Ra-Hl PyV identified in Rh. affinis was named

194 Rhinolophus affinis polyomavirus 5 (the fifth PyV discovered in Rh. affinis), and the Ra-Hl PyV

195 identified in H. larvatus was named Hipposideros larvatus polyomavirus 1. Similarly, the Ra-Mh

196 PyV identified in Rh. affinis was named Rhinolophus affinis polyomavirus 3, and the Ra-Mh PyV

197 identified in My. horsfieldii named Myotis horsfieldii polyomavirus 3.

198 Among the newly identified single-host PyVs (Fig 3), 19 PyVs shared 68-83\% nt sequence

199 identities with the closest-known species or with each other in the LTAg coding sequences,

200 therefore were considered to be novel PyV species according to a demarcation standard based on

$201 \geq 15 \%$ genetic divergence in the LTAg gene [36]. Seven PyVs shared $86-90 \%$ identity with the

202 closest known species or with each other in the LTAg gene, therefore showing insufficient

203 divergence; however, all were identified for the first time in their specific bat hosts. Furthermore,

204 these PyVs were exclusively detected in bat species separated by considerable distances $(\geq 564 \pm$

$205328 \mathrm{~km}$ ) (Fig 3 and S2 Table). They could therefore be considered novel PyV species, a proposal

206 further reinforced by additional correlation provided by the genetic selection analysis described

207 below [36]. Ra-Hl PyV and Ra-Mh PyV, exhibited 66\% and 77\% nt sequence identities in their

208 LTAg coding sequences with the closest known PyV, could therefore be proposed as novel PyV

209 species. However, the nomenclature for these PyVs requires further discussion as inter-family

210 host-switching is unprecedented for mammalian PyVs [36]. 
Table 2. Novel bat PyV genomes identified in Chinese bat hosts

\begin{tabular}{|c|c|c|c|c|c|c|c|c|c|c|c|c|c|c|c|c|}
\hline \multicolumn{4}{|c|}{ Host } & \multicolumn{5}{|c|}{ Polyomavirus } & \multirow[b]{2}{*}{ Length } & \multirow[b]{2}{*}{ GC \% } & \multirow[b]{2}{*}{ Genus } & \multicolumn{5}{|c|}{ Polyomavirus protein (a.a.) } \\
\hline No. & Sample Name & Year & Province & Colony ${ }^{\mathrm{a}}$ & Cytb closest match & Similarity \% ${ }^{\mathrm{b}}$ & Accession No. & Names of polyomavirus & & & & LTAg & STAg & VP1 & VP2 & $\mathrm{VP3}$ \\
\hline$\overline{1}$ & PyVG-NJ21C & 2016 & Yunnan & 2 & Aselliscus stoliczkanus (KU573085) & 98.9 & LC426669 & Aselliscus stoliczkanus polyomavirus 1 & 5,245 & 42.7 & Alphapolyomavirus & 656 & 189 & 371 & 311 & 242 \\
\hline 2 & PyV23-FCC16 & 2016 & Guangxi & 16 & Hipposideros larvatus (JN247027) & 96.3 & LC426670 & Hipposideros larvatus polyomavirus 1 & 5,266 & 42.4 & Alphapolyomavirus & 792 & 187 & 440 & 230 & 161 \\
\hline 3 & PyV15-NMC25 & 2016 & Guangxi & 14 & Miniopterus schreibersii (AY208138) & 99.3 & LC426671 & Miniopterus schreibersii polyomavirus 3 & 5,241 & 42.3 & Betapolyomavirus & 678 & 174 & 366 & 341 & 265 \\
\hline 4 & PyV14-NMC23 & 2016 & Guangxi & 14 & Miniopterus schreibersii (AY208138) & 99.0 & LC426672 & Miniopterus schreibersii polyomavirus 4 & 4,926 & 42.2 & Alphapolyomavirus & 740 & 195 & 402 & 237 & 192 \\
\hline 5 & PyV4-LPAC33 & 2015 & Guangxi & 11 & Myotis davidii (KM233172) & 94.7 & LC426673 & Myotis davidii polyomavirus 1 & 4,883 & 42.7 & Alphapolyomavirus & 744 & 190 & 397 & 225 & 190 \\
\hline 6 & PyV35-SWBC1 & 2016 & Fujian & 24 & Myotis horsfieldii (MF 143494) & 97.4 & LC426674 & Myotis horsfieldii polyomavirus 1 & 5,066 & 40.0 & Betapolyomavirus & 656 & 162 & 361 & 350 & 256 \\
\hline 7 & PyV33-SWBC37 & 2016 & Fujian & 24 & Myotis horsfieldii (MF143494) & 9.59 & LC426675 & Myotis horsfieldii polyomavirus 2 & 5,026 & 40.0 & Alphapolyomavirus & 684 & 187 & 367 & 331 & 231 \\
\hline 8 & PyV19-SWBC1 & 2016 & Fujian & 24 & Myotis horsfieldii(MF143494) & 97.4 & LC426676 & Myotis horsfieldii polyomavirus 3 & 5,033 & 40.4 & Betapolyomavirus & 684 & 83 & 365 & 325 & 204 \\
\hline 9 & PyV8-SHZC27 & 2016 & Xinjiang & 29 & Pipistrellus pipistrellus (AJ504443) & 95.4 & LC426677 & Pipistrellus pipistrellus polyomavirus 1 & 4,873 & 40.9 & Alphapolyomavirus & 722 & 188 & 403 & 270 & 239 \\
\hline 10 & PyV22-DXC12 & 2016 & Guangxi & 17 & Rhinolophus affinis (DQ297582) & 96.6 & LC426678 & Rhinolophus affinis polyomavirus 1 & 5,097 & 41.4 & Alphapolyomavirus & 695 & 189 & 369 & 311 & 157 \\
\hline 11 & PyVB-FN02C & 2016 & Yunnan & 1 & Rhinolophus affinis (DQ297582) & 97.1 & LC426679 & Rhinolophus affinis polyomavirus 1 & 5,097 & 41.4 & Alphapolyomavirus & 695 & 189 & 369 & 311 & 182 \\
\hline 12 & PyVB-FN04C & 2016 & Yunnan & 1 & Rhinolophus affinis (DQ297582) & 97.1 & LC426680 & Rhinolophus affinis polyomavirus 1 & 5,097 & 41.4 & Alphapolyomavirus & 679 & 189 & 369 & 311 & 194 \\
\hline 13 & PyVC-FN15C & 2016 & Yunnan & 1 & Rhinolophus affinis (DQ297582) & 97.2 & LC426681 & Rhinolophus affinis polyomavirus 2 & 5,224 & 43.5 & Alphapolyomavirus & 674 & 190 & 371 & 311 & 196 \\
\hline 14 & PyV19-DXC32 & 2016 & Guangxi & 17 & Rhinolophus affinis (DQ297582) & 96.8 & LC426682 & Rhinolophus affinis polyomavirus 3 & 5,033 & 40.4 & Betapolyomavirus & 654 & 163 & 365 & 325 & 204 \\
\hline 15 & PyV19-FCC38 & 2016 & Guangxi & 16 & Rhinolophus affinis (DQ297582) & 97.2 & LC426683 & Rhinolophus affinis polyomavirus 3 & 5,033 & 40.4 & Betapolyomavirus & 654 & 163 & 365 & 325 & 204 \\
\hline 16 & PyV19-SWAC9 & 2016 & Fujian & 24 & Rhinolophus affinis (D & 97.1 & 26684 & Rhinolophus affinis polyomavirus 3 & 5,032 & 40.5 & Betapolyomavirus & 663 & 163 & 365 & 325 & 204 \\
\hline 17 & PyV19-XDAC25 & 2016 & Fujian & 23 & Rhinolophus affinis (DQ297582) & 97.1 & LC426685 & Rhinolophus affinis polyomavirus 3 & 5,032 & 40.4 & Betapolyomavirus & 663 & 163 & 365 & 325 & 204 \\
\hline 18 & PyV19-XDAC40 & 2016 & Fujian & 23 & Rhinolophus affinis (DQ297582) & 97.1 & LC426686 & Rhinolophus affinis polyomavirus 3 & 5,032 & 40.5 & Betapolyomavirus & 663 & 163 & 365 & 325 & 204 \\
\hline 19 & PyVA-FN01C & 2016 & Yunnan & 1 & Rhinolophus affinis (DQ297582) & 97.1 & LC426687 & Rhinolophus affinis polyomavirus 3 & 5,032 & 40.4 & Betapolyomavirus & 683 & 163 & 365 & 325 & 204 \\
\hline 20 & PyVA-FN06C & 2016 & Yunnan & 1 & Rhinolophus affinis (DQ297582) & 97.2 & LC426688 & Rhinolophus affinis polyomavirus 3 & 5,032 & 40.5 & Betapolyomavirus & 683 & 163 & 365 & 325 & 204 \\
\hline 21 & PyV21-DXC30 & 2016 & Guangxi & 17 & Rhinolophus affinis (DQ297582) & 97.2 & LC426689 & Rhinolophus affinis polyomavirus 4 & 4,983 & 39.8 & Betapolyomavirus & 656 & 166 & 359 & 324 & 203 \\
\hline 22 & PyV21-FCC23 & 2016 & Guangxi & 16 & Rhinolophus affinis (DQ297582) & 97.2 & 26690 & Rhinolophus affinis polyomavirus 4 & 4,983 & 39.8 & Betapolyomavirus & 656 & 166 & 359 & 324 & 203 \\
\hline 23 & PyVD-FN16C & 2016 & Yunnan & 1 & Rhinolophus affinis (D & 97.2 & 26691 & us affinis polyomavirus 4 & 4,983 & 39.8 & Betapolyomavirus & 656 & 166 & 359 & 324 & 203 \\
\hline 24 & PyV23-DXC17 & 2016 & Guangxi & 17 & Rhinolophus affinis (DQ297582) & 97.1 & LC426692 & Rhinolophus affinis polyomavirus 5 & 5,266 & 42.4 & Alphapolyomavirus & 792 & 187 & 440 & 230 & 161 \\
\hline 25 & PyV11-DZ11C & 2016 & Zhejiang & 26 & Rhinolophus ferrumequinum kora & 97.2 & & us ferrumequinum polyomavirus 1 & 5,072 & 39.8 & Alphapolyomavirus & 650 & 193 & 368 & 310 & 195 \\
\hline 26 & PyV13-DZ26C & 2016 & Zhejiang & 26 & Rhinolophus ferrumequinum korai (JN392460) & 97.2 & LC426694 & Rhinolophus ferrumequinum polyomavirus 2 & 5,194 & 40.4 & Alphapolyomavirus & 688 & 189 & 370 & 311 & 207 \\
\hline 27 & PyV12-DZ13C & 2016 & Zhejiang & 26 & Rhinolophus ferrumequinum korai (JN392460) & 97.2 & LC426695 & Rhinolophus ferrumequinum polyomavirus 3 & 5,019 & 40.0 & Betapolyomavirus & 662 & 165 & 359 & 324 & 203 \\
\hline 28 & PyV12-DZ15C & 2016 & Zhejiang & 26 & Rhinolophus ferrumequinum korai (JN392460) & 97.2 & LC426696 & Rhinolophus ferrumequinum polyomavirus 3 & 5,019 & 40.0 & Betapolyomavirus & 661 & 165 & 359 & 324 & 203 \\
\hline 29 & PyVN-MS09C & 2016 & Yunnan & 4 & Rhinolophus pearsonii (KU531359) & 98.2 & LC426697 & Rhinolophus pearsonii polyomavirus 1 & 5,093 & 40.3 & Alphapolyomavirus & 660 & 196 & 369 & 311 & 242 \\
\hline 30 & PyVM-MS09C & 2016 & Yunnan & 4 & Rhinolophus pearsonii (KU531359) & 98.2 & C426698 & Rhinolophus pearsonii polyomavirus 2 & 5,225 & 41.0 & Alphapolyomavirus & 641 & 190 & 370 & 311 & 236 \\
\hline 31 & PyV28-YSC3 & 2016 & Fujian & 21 & Rhinolophus sinicus sinicus (KP257597) & 98.6 & LC426699 & Rhinolophus sinicus polyomavirus 1 & 5,443 & 44.4 & Alphapolyomavirus & 807 & 187 & 477 & 232 & 180 \\
\hline 32 & PyV29-YSC17 & 2016 & Fujian & 21 & Rhinolophus sinicus sinicus (KP257597) & 98.8 & LC426700 & Rhinolophus sinicus polyomavirus 2 & 5,529 & 44.7 & Alphapolyomavirus & 897 & 187 & 502 & 229 & 156 \\
\hline 33 & PyVE-NJ04C & 2016 & Yunnan & 2 & Rhinolophus sp. 2 KS2008 (EU434942) & 99.6 & LC426701 & Rhinolophus sp. CN 2016 polyomavirus 1 & 5,037 & 41.1 & Alphapolyomavirus & 662 & 189 & 372 & 311 & 243 \\
\hline 34 & PyVO-MS02C & 2016 & Yunnan & 4 & Rhinolophus tho & 98 & 6702 & Rhinolophus thomasi polyomavirus 1 & 5,032 & 40.0 & Betapolyomavirus & 664 & 165 & 362 & 325 & 254 \\
\hline 35 & PyVL-MS05C & 2016 & Yunnan & 4 & Rhinolophus thomasi (KY124333) & 98.6 & LC426703 & Rhinolophus thomasi polyomavirus 2 & 5,044 & 40.5 & Betapolyomavirus & 660 & 169 & 362 & 324 & 203 \\
\hline 36 & PyVP-NJ33C & 2016 & Yunnan & 3 & Rousettus leschenaultii ( & 99.9 & 126704 & Rousettus leschenaulti polyomavirus 1 & 5,007 & 42.8 & Betapolyomavirus & 665 & 162 & 364 & 338 & 216 \\
\hline 37 & PyVH-NJ32F & 2016 & Yunnan & 3 & Rousettus leschenaultii (DQ888669) & 99.1 & LC426705 & Rousettus leschenaulti polyomavirus 2 & 5,051 & 42.4 & Betapolyomavirus & 665 & 164 & 362 & 335 & 202 \\
\hline 38 & PyV24-LSC2 & 2016 & Guangxi & 18 & Scotophilus kuhlii (EF543860) & 100.0 & LC426706 & Scotophilus kuhlii polyomavirus 1 & 4,869 & 40.6 & Alphapolyomavirus & 718 & 191 & 392 & 241 & 196 \\
\hline 39 & PyV2-YSAC17 & 2015 & Guangxi & 9 & Scotophilus kuhlii (EF543860) & 99.9 & LC426707 & Scotophilus kuhlii polyomavirus 2 & 5,169 & 43.5 & Alphapolyomavirus & 818 & 188 & 465 & 218 & 160 \\
\hline 40 & PyV1-YHAC9 & 2015 & Guangxi & 10 & Scotophilus kuhlii (EF543860) & 100.0 & LC426708 & Scotophilus kuhlii polyomavirus 3 & 4,908 & 39.5 & Alphapolyomavirus & 752 & 187 & 396 & 238 & 193 \\
\hline 41 & PyV1-YSAC16 & 2015 & Guangxi & 9 & ophilus kuhlii (EF543860) & 100.0 & LC426709 & Scotophilus kuhlii polyomavirus 3 & 4,991 & 39.6 & Alphapolyomavirus & 736 & 187 & 396 & 238 & 193 \\
\hline 42 & PyV16-LZC25 & 2016 & Guangxi & 15 & Taphozous melanopogon (EF584221) & 93.2 & LC426710 & Taphozous melanopogon polyomavirus 1 & 5,133 & 41.5 & Alphapolyomavirus & 711 & 190 & 377 & 310 & 193 \\
\hline
\end{tabular}

213 a Colony numbers. The locations of colonies are shown in Fig 1, with detailed information in S1 Table.

214 b Similarity in percentage of amplified bat cytb genes with the closest GenBank hit. The GenBank accession numbers of amplified bat

$215 c y t b$ genes are shown in S4 Table. 


\section{Phylogenetic analysis and identification of host-switching events.}

217 In order to verify the host-switching events, an LTAg amino acid tree was constructed to 218 compare the 28 new PyVs with 126 known Alpha- and Betapolyomavirus species (including 38

219 bat-borne PyV reference species). As shown in Fig 4, 28 new bat PyVs (colored in the figure)

220 clustered within the Alphapolyomavirus $(\mathrm{n}=19)$ and Betapolyomavirus $(\mathrm{n}=9)$ genera along with

22138 known bat PyVs. Within each viral genus, bat PyV sequences grouped into 7 clusters, referred

222 to here as Alpha 1-7 and Beta 1-7, respectively. Among these novel PyVs, two viruses, Ra-H1

$223 \mathrm{PyV}$ in the Alphapolyomavirus and Ra-Mh PyV in the Betapolyomavirus genera, were identified

224 in bat hosts of different families living in the same colony, suggestive of two viral host-switching

225 events, labeled as host-switching event 1 and 2 (HS1 and 2 in Fig 4). Furthermore, a third PyV

226 clustered in the phylogenetic tree with PyVs carried by bats of different families (Hipposideridae

227 and Rhinolophidae), indicative of another inter-family host-switching event (HS3 in Fig 4).

228

The first host-switching event HS1 in the Alpha 3 cluster (Fig 4) occurred between Rh. affinis

229 (family Rhinolophidae) and H. larvatus (family Hipposideridae). Strikingly, Ra-H1 PyV,

230 characterized in Rh. affinis (Rhinolophus affinis polyomavirus 5) and H. larvatus (Hipposideros

231 larvatus polyomavirus 1), exhibited 100\% nt identity between both genomes. Importantly, they

232 were detected in multiple animals and, also, from different colonies. The Rhinolophus affinis

233 polyomavirus 5 was detected in two colonies (colonies 16 and 17 separated $10 \mathrm{~km}$ apart in south

234 Guangxi province in southern China) with detection rates of $1 / 22$ and 8/28, respectively. Notably,

235 the Hipposideros larvatus polyomavirus 1 was also detected in colonies 16 and 17 in H. larvatus,

236 also in Guangxi province, with positive rates of 3/19 and 2/10, respectively. 
245 horsfieldii polyomavirus 3 had a high positive rate $63.8 \%(23 / 36)$ in My. horsfieldii bats within a

246 single colony (colony 24 Fig 1 and S1 Table) where, notably, Rh. affinis and My. horsfieldii

247 species roosted together. In this cave, Rh. affinis also harbored Rhinolophus affinis polyomavirus

2483 with a 57\% (12/21) detection rate. Geographically, Rhinolophus affinis polyomavirus 3 was 249 widely distributed in $R h$. affinis hosts.

250 The third host-switching event, HS3, in the Alpha 7 cluster (Fig 4) occurred between $A$. 251 stoliczkanus (family Hipposideridae) and Rhinolophus spp. (family Rhinolophidae). The 252 Aselliscus stoliczkanus polyomavirus 1 exhibited $87 \%$ and $86 \%$ nt identity with Rhinolophus 253 affinis polyomavirus 2 and Rhinolophus pearsonii polyomavirus 2, respectively (Fig 3). The two 254 sampling locations of the Rhinolophus species were from caves located $731 \mathrm{~km}$ and $86 \mathrm{~km}$ 255 respectively from the cave in which the single Aselliscus stoliczkanus polyomavirus 1 positive 256 sample was detected.

257 Besides these three host-switching events, a PyV-positive rectal tissue sample, named 
Pipistrellus pipistrellus polyomavirus XJPp02 [strain PyV10-YXAC25], collected from a

Pipistrellus pipistrellus (family Vespertilionidae) bat in colony 28 in northwestern Xinjiang

exhibited 98\% nt identity in a partial VP1 fragment ( 270 nt) with Rattus norvegicus

polyomavirus 1 (detected in German rats) [35] in the Alphapolyomavirus genus (marked as

\section{Correlation of genetic divergences between PyVs and bat hosts.}

274 As shown in Fig 3 and 4, although seven new PyVs showed high LTAg nt identities (86-90\%)

275 with each other or the closest known PyVs, the phylogenetic clustering supported codivergence

276 events rather than host-switching events. For instance, Rhinolophus pearsonii polyomavirus 1 and

277 Rhinolophus affinis polyomavirus 1 shared 88\% nt identity (Alpha 6); Rhinolophus affinis 
279 Rhinolophus ferrumequinum polyomavirus 2 shared 89\% identity with Rhinolophus simulator

280 polyomavirus 1 (LC269978) previously identified in Zambian bats (Alpha 7); and Rhinolophus

281 ferrumequinum polyomavirus 1 shared 90\% identity with Rhinolophus simulator polyomavirus 3

282 (LC269980) also identified in Zambian bats (Alpha 6). These last two instances showed that

283 PyVs from Chinese and African horseshoe bats were remarkably similar despite the geographical

284 separation.

285 To investigate further the factors potentially influencing the divergence between PyVs with

high nt sequence identities, Mantel's correlation tests were performed to compare the divergence

among PyV genome sequences with those among $c y t b$ genes of their corresponding hosts and to

294 of these three groups of data showed that there was negligible correlation between geographical

295 distances and divergence for either hosts or viruses. Moreover, the geographical proximity

296 between the colonies had little effect on the co-evolution of viruses with their respective hosts.

297 Analysis using the first group of data showed that a significant correlation existed between

298 host and PyV divergence per genus considering the 42 genomes identified in China. The 
$\left.300<2 \times 10^{-4}\right)$ and $0.65\left(P<1.5 \times 10^{-3}\right)$, respectively. This first dataset supported the intra-host 301 divergence model of $\mathrm{PyV}$ and hosts, with multiple viral lineage duplications, in support of a 302 previous model of PyV evolution [17]. The addition of PyV sequences sampled from African 303 Rhinolophus spp. [26] increased the correlation index between host and viral divergence as the 304 number of considered sequences increased. Altogether, these findings support an intra-host 305 divergence model for the radiation of PyV species within the order Chiroptera. In the third group, 306 by removing 8 sequences (also marked as host switching lineages in Fig 4) in the Alpha- and 3 in 307 the Betapolyomavirus genera, which, notably, included host-switching events (HS1-HS3) from 308 both PyV genera, the correlation indices increased $40 \%$ and $30 \%$, respectively. 
Table 3. Correlation analyses of genetic divergences between PyVs and bat hosts.

\begin{tabular}{|c|c|c|c|c|c|c|c|c|}
\hline \multirow{2}{*}{\multicolumn{2}{|c|}{ Group of PyVs for analyzing }} & \multirow{2}{*}{$\begin{array}{l}\text { Number of } \\
\text { samples }\end{array}$} & \multicolumn{4}{|l|}{ Correlation } & \multicolumn{2}{|c|}{ Partial Correlation $^{\mathbf{b}}$} \\
\hline & & & Genus & Geography & Host & Geography| Host $^{\mathrm{a}}$ & Geography| Host & Host| Geography \\
\hline \multirow{2}{*}{\multicolumn{2}{|c|}{ Current study }} & 23 & $\begin{array}{l}\text { Alphapolyomavirus } \\
\mathrm{P} \text { value }\end{array}$ & $\begin{array}{l}0.05 \\
3.45 \mathrm{E}-01\end{array}$ & $\begin{array}{l}0.47 \\
2.00 \mathrm{E}-04\end{array}$ & $\begin{array}{l}0.15 \\
1.30 \mathrm{E}-01\end{array}$ & -0.02 & 0.47 \\
\hline & & 19 & $\begin{array}{l}\text { Betapolyomavirus } \\
\mathrm{P} \text { value }\end{array}$ & $\begin{array}{l}0.14 \\
1.09 \mathrm{E}-01 \\
\end{array}$ & $\begin{array}{l}0.65 \\
1.50 \mathrm{E}-03\end{array}$ & $\begin{array}{l}0.17 \\
7.59 \mathrm{E}-02\end{array}$ & 0.04 & 0.64 \\
\hline \multirow{2}{*}{\multicolumn{2}{|c|}{ Including Zambian Rhinolophus PyVs }} & 26 & $\begin{array}{l}\text { Alphapolyomavirus } \\
\text { P value }\end{array}$ & $\begin{array}{l}-0.13 \\
9.00 \mathrm{E}-01\end{array}$ & $\begin{array}{l}0.48 \\
2.00 \mathrm{E}-04\end{array}$ & $\begin{array}{l}-0.10 \\
8.09 \mathrm{E}-01\end{array}$ & -0.10 & 0.48 \\
\hline & & 22 & $\begin{array}{l}\text { Betapolyomavirus } \\
\text { P value }\end{array}$ & $\begin{array}{l}0.22 \\
1.09 \mathrm{E}-01\end{array}$ & $\begin{array}{l}0.65 \\
3.00 \mathrm{E}-04\end{array}$ & $\begin{array}{l}0.07 \\
2.80 \mathrm{E}-01\end{array}$ & 0.23 & 0.66 \\
\hline Removing & $\begin{array}{l}\text { Aselliscus stoliczkanus polyomavirus } 1 \text { [PyVG-NJ21C] } \\
\text { Hipposideros larvatus polyomavirus } 1 \text { [PyV23-FCC16] } \\
\text { Rhinolophus sinicus polyomavirus } 1 \text { [PyV28-YSC3] } \\
\text { Rhinolophus sinicus polyomavirus } 2 \text { [PyV29-YSC17] } \\
\text { Scotophilus kuhlii polyomavirus } 1 \text { [PyV24-LSC2] } \\
\text { Scotophilus kuhlii polyomavirus } 2 \text { [PyV2-YSAC17] } \\
\text { Taphozous melanopogon polyomavirus 1 [PyV16-LZC25] } \\
\text { Rhinolophus affinis polyomavirus } 5 \text { [PyV23-DXC17] }\end{array}$ & 18 & $\begin{array}{l}\text { Alphapolyomavirus } \\
\mathrm{P} \text { value }\end{array}$ & $\begin{array}{l}-0.10 \\
7.49 \mathrm{E}-01\end{array}$ & $\begin{array}{l}0.82 \\
1.00 \mathrm{E}-04\end{array}$ & $\begin{array}{l}-0.08 \\
7.02 \mathrm{E}-01\end{array}$ & -0.05 & 0.82 \\
\hline Removing & $\begin{array}{l}\text { Myotis horsfieldii polyomavirus 3 [PyV19-SWBC1] } \\
\text { Rhinolophus simulator polyomavirus } 4 \text { (LC269981) } \\
\text { Rhinolophus blasii polyomavirus } 1 \text { (LC269976) }\end{array}$ & 19 & $\begin{array}{l}\text { Betapolyomavirus } \\
\text { P value }\end{array}$ & $\begin{array}{l}0.01 \\
2.48 \mathrm{E}-01\end{array}$ & $\begin{array}{l}0.9 \\
1.00 \mathrm{E}-04\end{array}$ & $\begin{array}{l}0.08 \\
2.39 \mathrm{E}-01\end{array}$ & -0.14 & 0.9 \\
\hline
\end{tabular}

$310{ }^{a}$ Correlation between evolutionary distance among hosts and geographical distances of their detected location

311 b Partial correlation controlling for the effect of the variable after the vertical bar 


\section{Bayesian estimation of tMRCA of bat hosts and PyVs.}

313 To test whether the host-switching events of the PyV species occurred after the divergence of

314 host species, the times to most recent common ancestor (tMRCA) of both bat hosts and the PyVs

315 were estimated employing a Bayesian approach. As shown in Fig 5A, the tMRCA of bat hosts

316 were estimated based on the cytb sequences $(\sim 1800 \mathrm{bp})$ of bats that harbored the 28 novel PyVs.

317 The results showed that divergence among Rhinolophus species happened 14.2 to 17.3 million

318 years ago (MYA) with a 95\% highest posterior density (HPD), which is consistent with

319 previously reported divergence times (16-17 MYA) of the bat hosts based on mitochondrial

320 DNA [39] and other sampled bat families [40]. The Bayesian results for PyV divergence times

321 (Fig 5B) also showed that the tMRCAs for both Alpha- and Betapolyomavirus genera were

115-182 MYA with 95\% HPD, overlapping with the divergence times of the tMRCA of placental

mammals (92 to $116 \mathrm{MYA}$ ) [41]. However, when compared with the Bayesian results for the bat

hosts, the time scale of PyV divergence was not always in agreement: specifically, 1) the

divergence time between Rhinolophus and Hipposideros hosts was estimated with a median 36.3

326 MYA in the 95\% HPD range $(34,38)$ MYA, while the tMRCAs of Rhinolophus affinis

327 polyomavirus 5 and Hipposideros larvatus polyomavirus 1 were estimated with a median of 0.02

328 MYA in the 95\% HPD range $(0.0012,0.12)$ MYA; 2) The tMRCAs of Myotis horsefieldii

polyomavirus 3 and Rhinolophus affinis polyomavirus 3 had a median of 1.05 MYA in the 95\%

HPD range $(0.4,1.9)$ MYA, while the median tMRCA of both corresponding host species was 
333 in the 95\% HPD range $(6.6,17.8)$ MYA, however, the tMRCA between both hosts had a median

334 of 36.3 MYA in the 95\% HPD range (34.3, 38.2) MYA. Finally, 4) the estimated time of

335 divergence between Scotophillus kuhlii polyomavirus 2 and Pipistrellus pipistrellus polyomavirus

336 1, with a median of 37 MYA in the 95\% HPD range $(23,54)$ MYA, was less than the predicted

337 divergence time between the hosts, with a median of 44.42 MYA in the 95\% HPD range $(40,49)$

338 MYA. In the four examples, the most parsimonious explanation for the lesser median ages to

339 divergence of the PyVs compared to the longer tMRCAs for the bat hosts is that these represent 340 examples of inter-family viral host-switching events.

Intra-lineage host duplication and host-switching events during bat PyV evolution.

343 The findings of the Mantel test and other studies [17] supporting intra-host divergence for PyVs,

344 required reconciliation with the compelling evidence of similar viral species infecting

345 evolutionarily distant bat species. Co-phylogenetic reconstruction analyses using JANE 4 were

346 therefore performed separately for both viral genera. The best-supported solution to explain the

347 divergence in the genus Alphapolyomavirus for the novel Chinese PyVs and African samples was

348 modeled with 11 co-speciations, 4 lineage duplications, 13 lineage losses and 9 host-switching

349 events (Fig 6A). Among the 9 inferred host-switching events, in agreement with the tMRCA

350 estimations, it was implied that Rhinolophus affinis polyomavirus 5 originated in the lineage

351 which gave rise to Hipposideros larvatus polyomavirus 1, Rhinolophus sinicus polyomavirus 1

352 and 2, and Scotophilus kuhli polyomavirus 2 indicating that Rh-Hl PyV was originally a PyV

353 harbored by $R h$. affinis, which was subsequently transmitted to H. larvatus. The best-supported 
solution also suggested that the lineage of Rhinolophus affinis polyomavirus 2 had given rise to

Aselliscus stoliczkanus polyomavirus 1. Other host-switching events inferred earlier in the

divergence among PyVs were more difficult to corroborate with the available tMRCA data.

although the presence of duplication events could explain the existence of more than one

\section{Positive selection in divergence of Chinese and African Rhinolophus PyVs are focused on}

\section{the external surface of the VP1.}

370 Given the diversity of viral species identified in the genus Rhinolophus, viral proteins under

371 positive selection during the evolution of the different PyV lineages were of interest as possible

372 speciation determinants. We performed a Bayesian analysis to calculate the $\omega$ rate $(\mathrm{dN} / \mathrm{dS})$ to

373 identify genomic regions of the Alpha- and Betapolyomavirus genera in Rhinolophus PyV

374 clusters, which were likely subject to positive selection (S3 Fig). As expected, the majority of the 
horseshoe bat PyV genomes were found to be under neutral or negative selection $(\omega$ values $\leq 1)$; however, a number of residues in VP2, VP1, LTAg and STAg proteins did show evidence of

377 positive selection ( $\omega$ values $>1)$ in both viral genera (shown in red in S3 Fig) and amino acid 378 residues relative to human BK virus (species of Human polyomavirus 1) and simian virus 40 379 (species of Macaca mulatta polyomavirus 1) are detailed in S5 Table. We focused on the major capsid protein VP1 as this determines antigenicity and the engagement of sialylated glycans present on host glycoproteins and glycolipids during cellular attachment and entry in mammalian 382 (murine, human and monkey) viruses [42-46].

383 In the Rhinolophus alphapolyomavirus cluster, 11 residues were found under positive selection, 384 located in four regions of VP1 (S5 Table): in the BC-loop (D77, N79 and V82), the DE-loop 385 (L135, A139 and T143), the HI loop (N274, N275 and S279) and the C-terminal residues I367 and N369. In the Rhinolophus betapolyomavirus cluster, 9 residues had $\omega$ values $>1$ at the 387 N-terminus (K17), in the BC loop (A75, A77, T80, V82 and P84), the DE loop (N134 and Q149) and the EF loop (S178). No positively selected amino acids were identified in the interior of the 
Discussion

391 While mammalian PyVs are highly host specific [12] there are data suggesting cross-species

jumps of PyVs between closely related mammalian hosts. Previously, short-range intra-genus

host-switching events of PyVs were identified in African horseshoe bats, occurring between $R h$.

394 simulator and Rh. blasii in a single Zambian cave [26]. Strikingly, the genomes of PyVs

395 characterized in respective bat species were $99.9 \%$ identical and contained only four virus

396 species-specific polymorphisms. Importantly, these polymorphisms were found to be present in

397 both spleen and kidney samples of each bat host species, indicating a systemic and productive

398 infection. The genetic stability and restricted host-specificity of mammalian PyVs had previously

399 suggested a model of strict codivergence for PyV evolution [47]. However, this is inconsistent

400 with family-wide patterns of diversity, where an important contribution of intra-host lineage

401 duplication, recombination and, possibly, host-switching events is evident. A model of intra-host

402 divergence, with these additional evolutionary processes, occurring over the ancient timescales

403 for association of PyVs with multicellular life, has been proposed to explain the extant diversity

404 within the family Polyomaviridae [17]. The findings in the present study extend these

405 observations to PyVs in Chinese horseshoe bats and other bat hosts (see model in Fig 7) and

406 provide the first definitive evidence of inter-family host-switching of PyVs in mammals

407 occurring among the bat families Rhinolophidae, Hipposideridae and Vespertilionidae.

408 The host-switching events of PyVs identified in the present study indicate that transmission of

409 PyVs between different bat species has been an ongoing process during the evolutionary history

410 of PyVs. HS1 and HS2 occurred between bats of different families and strains of PyVs sharing 
$411100 \%$ or $97-100 \%$ nt identity were characterized in their respective hosts. The tMRCA analysis

412 estimated that HS1 and HS2 occurred between 0.02-1.05 MYA. Considering the "slow"

413 evolutionary rate of PyVs $\left(8 \times 10^{-9}\right.$ substitutions per site per year as previously estimated [48]),

414 the transmission events of Rh-Hl PyV and Rh-Mh PyV in bats happened recently, with a small

415 number of mutations accumulating in the viral genomes during viral adaptation to the permissive

416 hosts. Compared with HS1 and HS2, HS3 was estimated to have occurred much earlier, between

417 6.6-17.8 MYA, with the Aselliscus stoliczkanus polyomavirus 1 showing $87 \%$ nt identity with

418 the closest PyV (Rhinolophus affinis polyomavirus 2), indicating that the former had been

419 transmitted from Rhinolophus bats to A. stoliczkanus bats for a long period of time, had

420 co-evolved with A. stoliczkanus and, finally, had formed a novel PyV species. In addition to

421 these observed host switching events, the tMRCA estimation and correlation analysis showed

422 strikingly that numerous PyVs had also been derived from host switching, thereby shaping the

423 evolutionary history of PyVs and providing evidence that inter-species transmission of PyVs, at

424 least in bats, is not as rare as previously thought.

425 An issue raised by the present study is how to define and name a PyV species when

426 inter-family host-switching events of PyV are identified. The ICTV criteria for the formal

427 designation of PyV species in mammals must include the binomial name of the host species [36];

428 nevertheless, the inter-family host-switching events of PyVs discovered in the present study as

429 well as the inter-species host-switching event also identified in Rhinolophus hosts in a previous

430 study [26] which has challenged this nomenclature system for bat PyVs. Furthermore, numerous 
431 host-switching lineages have also been characterized here by correlation analyses and dating

432 estimation. All these findings suggest that host-switching of PyVs has occurred not only in

433 different bat species but also at different time points in different geographical locations. The

434 ICTV nomenclature criteria should therefore be updated to include recognition of PyVs derived

435 from two different mammalian host species.

436 Inter-species transmission has already been observed in bird PyVs such as budgerigar

437 fledgling disease PyV (BFDV), which can infect multiple avian species with pronounced

438 pathogenicity [49]. Nevertheless, the ICTV nomenclature has utilized only the order level of the

439 host species in the naming scheme: for instance, species belonging to BFDV have been classified

440 as Aves polyomavirus 1. This naming scheme could also be expanded using the more descriptive

441 host species, as with the mammalian PyV species. So far, PyVs with host-switching events have

442 only involved two host species, illustrating the limited ability of PyVs for transmission between

443 different mammalian hosts. Therefore, the improved naming scheme of novel PyVs with

444 host-switching events proposed here would include the binomial names of both host species; e.g.,

445 Rhinolophus affinis-Hipposideros larvatus polyomavirus (Rh-Hl PyV), identified in the present

446 study, would become the prototype of a new species named Rhinolophus affinis-Hipposideros

447 larvatus polyomavirus 1.

448 Another ICTV criterion is the allowable genetic distance of the LTAg coding sequence $(\geq$

449 15\%) to demarcate PyV species. This cut-off value is not strict, as exemplified by two PyVs

450 identified in two different species of squirrel monkeys which shared $89 \%$ sequence identity but 
451 were recognized as two separated PyV species [36]. In the present study, we have discovered numerous novel PyVs carried by diverse bat species, all exhibiting 86-90\% sequence identities with their closest known species or with each other. These should be considered new PyV species since all were detected exclusively in distinct bat species in different areas and exhibiting strict host specificity. Examples include the PyVs identified in different Rhinolophus bat species from

456 China and Africa sharing $89-90 \%$ nt identify. The correlation and genetic selection analysis 457 reported here has shown that the high sequence identity of different PyVs carried by different 458 host species was due to the co-divergence with strong negative selection of PyVs in respective 459 hosts, indicating that the $\geq 15 \%$ cut-off in LTAg gene identity for species demarcation is perhaps 460 no longer adequate and should be updated to $\geq 10 \%$. With regard to the relationship between 461 Chinese and African bat PyVs referred to above, it is of interest to note that hepadnaviruses 462 (pararetroviruses with DNA genomes) identified in Chinese Rhinolophus bats in another study [49] were also found to exhibit the closest genetic relation (79.6-79.7\% nt identify) with viruses carried by African Rhinolophus bats, [50] thereby suggesting that co-divergence of DNA viruses within their Rhinolophus bat hosts is relatively common.

467 finding that has considerably increased our knowledge of both the genomic diversity and the 468 evolution of PyVs. Additionally, since there is a dearth of commercially available, standardized 469 bat cell lines for isolation and identification of bat-borne viruses there are now added options to 470 establish immortalized bat cell lines from many bat species by using LTAg genes cloned from 471 divergent PyVs identified in specific Chinese bat species. At present, the Tb1 Lu cell line derived 
473 line available in the American Type Culture Collection (ATCC Nr. CCL-88) [51]. Recently,

474 however, Banerjee et al. [52] have reported immortalization of primary bat kidney cell lines from

475 the big brown bat (Eptesicus fuscus, family Vespertilionidae) which were stably transformed by a

476 plasmid harboring a little brown bat (Myotis lucifugus, family Vespertilionidae) PyV LTAg. The

477 ability to establish cell lines from different tissues of diverse bat species and to make these

478 available to researchers is a prerequisite for replication studies, virus-host interactions and new

479 virus discoveries.

480 Papillomaviruses (PVs), which are closely related to PyVs and share similar properties such

481 as, high genetic stability and strict host specificity, have been shown to cause transient and

482 potentially cross-species infections. García-Pérez et al. [53] reported that PVs do not follow strict

483 host restrictions in free-ranging Iberian bats (Eptesicus serotinus, family Vespertilionidae), as

484 they found evidence of host-switching events between two different bat species (E. serotinus and

485 E. isabellinus). They therefore recommended a revised paradigm of strict host specificity in PVs $486 \quad[53]$.

487 The findings presented here have possible implications for the study of zoonotic transmissions 488 involving highly virulent viral pathogens in bats. For example, horseshoe bats within the family

489 Rhinolophidae have been found to harbor genetically diverse severe acute respiratory syndrome

490 (SARS)-like corona viruses, which acted as major reservoirs for spillover of SARS coronavirus

491 to humans [7]. The Middle Eastern respiratory syndrome (MERS) coronavirus has similarly been

492 associated with severe disease and lethal respiratory infections, mostly in Saudi Arabia. Likely 
transmitted via dromedary camels, it has been shown to be closely related to several bat

494 coronaviruses, including those sequenced from species within the family Vespertilionidae

495 (Neoromicia capensis, Pipistrellus abramus and Vespertilios uperans) [54]. Recently, swine

496 acute diarrhea syndrome (SADS) coronavirus has been associated with high morbidity and

497 mortality in pig populations in Guangdong province, China in close proximity to the origin of the

498 SARS pandemic, and striking similarities between the SADS and SARS outbreaks in

499 geographical, temporal, ecological and etiological settings exist [9]. We consider noteworthy that

500 in the present study we identified host-switching events of the normally highly host-restricted

501 PyVs within the bat families Rhinolophidae and Vespertilionidae. Whether this reflects a greater

502

503

504

505

506

507

508

509

510

511

512

neuroendocrine carcinoma of the elderly. BoPyV3, is also related to human polyomavirus 6

513

propensity for host-switching in these bat families when compared with others and what mechanisms are involved require further functional studies. Conceivably, given the high

prevalence we and others have detected of bat PyVs, co-infection of bat species within the families Rhinolophidae and Vespertilionidae may affect the prevalence and/or transmission of CoVs and other high virulent pathogens in bats. This suggestion warrants further study.

Our findings that inter-family transmission of PyVs can occur in mammals may also have implications for studies of viral oncogenesis proposed previously for the Polyomaviridae. For example, three bovine PyVs (BoPyVs) have been identified in cattle [55] with more likely given the 13 so far identified as infectious agents in humans [56]. Intriguingly, BoPyV2, occupies the same clade as Merkel cell PyV which is causally implicated in oncogenic transformation in a which has been reported in non-melanoma skin cancers, specifically keratocanthomas [57]. 
514 Colorectal cancer has been suggested to have an infectious disease etiology [58] and the

515 relatively high thermoresistance of PyVs may preclude inactivation by lowered cooking

516 temperatures [59]. Similarly, in field studies, sequences of BK virus were identified by

517 metagenomics in the feces of non-human primates [60]. Our evidence that inter-family

518 transmission of PyVs can occur in bats provides a precedent for studies of longer-range

519 transmission of PyVs in mammals.

520 In addition to three bat inter-family host-switching events of PyVs, we also identified a partial

521 VP1 fragment in rectal tissue of a $P$. pipistrellus bat collected in northwestern Xinjiang that

522 showed $98 \%$ nt identity with the VP1 of a previously described Rattus norvegicus polyomavirus

5231 identified in Germany [35]. Further qPCR assay also detected the virus in lung tissue of another

$524 P$. pipistrellus bat but not from any brown rat $(\mathrm{n}=51)$ collected in the same Xinjiang area. This

525 preliminary observation provides an impetus to investigate potential PyV host-switching between

526 mammal species beyond families.

527 The host-switching events identified in this study were strongly associated with the ecological

528 environment of the hosts, with host-switching only identified between different bat species in one

529 to two colonies. Our study suggests that PyVs can transmit between different mammalian hosts

530 between two different animal families by frequent contact of hosts within specific environments,

531 such as the densely packed bat populations that occur in large hibernacula in caves. The

532 host-switching PyVs were identified from rectal tissues of multiple individuals, indicating that

533 shedding via feces is a key factor for viral transmission by the fecal-oral route between different

534 host species. 
PyV attachment to target cells is mediated by cell surface receptors. MHC class I acts as a co-receptor and SV40 employs the ganglioside GM1 as receptor [61]. The finding that gangliosides are also primary receptors for human PyVs via VP1 [62] suggests that this may be a general property for primate PyVs although whether this is applicable to other mammalian PyVs, including bats, is unknown. In the present study, amino acid residues in VP1 were found to be

543 serological subtypes are assigned based on mutations present within the BC loop [63]. It has also

545 and binding to sialic acid moieties was attributed to amino acids residues within the BC, DE and

546 HI loops, suggesting that these VP1 domains play a key role in virus attachment and cellular

547 entry events [45]. The surface loops of VP1, i.e. the BC, DE, HI and EF loops, are responsible for

548 viral antigenicity and escape mutants cluster in these regions, suggesting they represent

549 conformational epitopes recognized by host neutralizing antibodies $[65,66]$. VP1 surface loops

550 form a unique virus-host interaction conformation that determines the host range, cellular tropism

551 and pathogenicity which may explain the enrichment of positive selected residues within these

552 regions [67]. The availability of annotated bat genomes in the future, as proposed by the genome

553 consortium Bat1K [68], and annotation of the genes encoding putative entry receptors (such as,

554 for example, the cell surface ganglioside and other sialylated glycan receptors) from within the

555 bat families Rhinolophidae, Hipposideridae and Vespertilionidae from species previously found 
556 to harbor identical or closely related viruses would facilitate the functional characterization of the

557 cellular receptors/attachment factors and of which amino acid residues in VP1 are required for

558 binding and entry. Availability of annotated bat genomes would also help determine whether

559 certain families of bats are more susceptible than others to virus-host switching events. 
Materials and methods

Ethics statement.

562

563

564

565

566

567 China.

568

569

570

571

572

573

574

575

576

577

578

collected in Yunnan province were pooled and subjected to viral metagenomic analysis, as per 579 our previously published method [34]. Due to the complexity of the PyV-related reads detected

580

The procedures for sampling bats and brown rats in this study were reviewed and approved by

the Administrative Committee on Animal Welfare of the Institute of Military Veterinary

Medicine (Laboratory Animal Care and Use Committee Authorization permits JSY-DW-2010-02

and JSY-DW-2016-02). All live animals were maintained and handled according to the Principles

and Guidelines for Laboratory Animal Medicine (2006), Ministry of Science and Technology,

\section{Sample information.}

The tissues of 1,083 bats in the present study were archived and sub-packed samples were stored at $-80^{\circ} \mathrm{C}$ following collection between 2015-2016 from 29 colonies distributed across the

provinces of Yunnan, Fujian and Zhejiang, Guangxi and Xinjiang in China. Archived samples of rectal and kidney tissues from brown rats $(n=51)$ were collected in Xinjiang between 2015-2016 [38]. Detailed information of sampled bats and rats is shown in S1 Table.

\section{Polyomavirus screening and complete genome sequencing.}

Approximately $50 \mathrm{mg}$ samples of rectal and lung tissues from the 208 bats in colonies 1-4 by the viral metagenomic analyses, the PyV-specific nested PCR protocol [69] based on the VP1 
gene developed by Johne et al. in 2005 [27] was employed to screen all metagenomic samples,

and partial VP1 amplicons were cloned and bidirectionally sequenced by standard methods. In

addition, the PCR was expanded to screen genomic DNA extracts from rectal tissues of bats

collected from other Chinese provinces.

585 Genomic DNA from each bat tissue sample was extracted manually using the TIANamp

586 Genomic DNA Kit (Tiangen), according to the manufacturer's protocol. PCR screening

587 employed the PCR master mix (Tiangen) with the following thermocycling conditions: 45 cycles

588 for both outer and inner PCRs with denaturation at $94^{\circ} \mathrm{C}$ for $30 \mathrm{~s}$, annealing at $46^{\circ} \mathrm{C}$ (outer PCR)

or $56^{\circ} \mathrm{C}$ (inner PCR) for $30 \mathrm{~s}$ or $1 \mathrm{~min}$, and extension at $72^{\circ} \mathrm{C}$ for $1 \mathrm{~min}$, with $\mathrm{ddH}_{2} \mathrm{O}$ as a

negative control. Positive PCR amplicons were purified by gel extraction (Axygen) and

ligated into vector pMD-18T (TaKaRa) and used to transform E. coli DH5 $\alpha$ competent cells

593 (Tiangen). Four clones from each VP1 amplicon were picked for Sanger sequencing. GenBank

594 accession numbers of 192 partial PyV VP1 sequences are showed in S3 Table.

595 Oligonucleotide primers for inverse PCRs for amplification of the $5 \mathrm{~kb}$ PyV genomes were

596 design based on the partial VP1 sequences. Multiple pairs of hemi-nested degenerate primers

597 were subsequently designed for VP1 clusters which showed close phylogenetic relationships (see

598 S6 Table). All $\sim \mathrm{kb}$ products were amplified from bat genomic DNA by inverse PCR employing

599 the PrimeSTAR MAX DNA polymerase (Takara), purified by gel extraction (Axygen), cloned

600 into pCR-Blunt II-TOPO vector (Invitrogen) and bidirectionally sequenced by primer walking to

601 Phred quality scores $>30$ by 3730xl DNA Analyzer (Genewiz). After removal of vector 
sequences, the full genomes of each PyV were assembled by Geneious software using the partial

$603 \mathrm{VP} 1$ and $5 \mathrm{~kb}$ products.

604 A real-time PCR (qPCR) method (S6 Table) was established based on a 59 nt fragment of the

605 VP1 genes of Pipistrellus pipistrellus polyomavirus XJPp02 and Rattus norvegicus polyomavirus

6061 was designed to screen spleen, kidney, lung, liver, rectal and brain tissues in all the $P$.

607 pipistrellus bats $(\mathrm{n}=158)$ collected in three locations in Xinjiang in 2016 and also rectal and

608 kidney tissues from brown rats $(n=51)$ available from collections in two other locations in

609 Xinjiang during 2015-2016 (S1 Table). The extracted DNAs from the tissues were amplified

610 using the Probe qPCR kit (TaKaRa) as per the manufacturer's protocol on a Stratagene

611 Mx3000P.

612

\section{Phylogenetic analysis.}

614 The pairwise identity of the LTAg coding sequences was calculated with Sequence Demarcation

615 Tool (SDT) v1.2 [70]. The nucleotide sequence of host $c y t b$, the sequences of PyV genes and

616 proteins were aligned using MAFFT under the algorithm FFT-NS-i [71]. Phylogenetic trees were

617 inferred using MrBayes v3.2, with $10^{6}$ generations chains to ensure $<0.01$ standard deviations

618 between the split frequencies and $25 \%$ of generations discarded as burn-in [72], as described

619 previously [73]. For the LTAg multiple-sequence alignment, a mixed substitution model was

620 used to explore the best substitution model when inferring the topology. On the other hand, the

621 best fitting nucleotide substitution models for host cytb and the PyV LTAg coding sequence

622 alignments were chosen with MEGA7 [74] by choosing the lowest Bayesian information 
623

624

625

626

627

628

629

630

631

632

633

634

635

\section{Correlation analysis.}

637 The divergence correlation between the hosts and PyVs in the Alpha- and Betapolyomavirus

criterion (BIC).

The tMRCA for hosts and viral samples were estimated with Bayesian analyses conducted as

4 independent MCMC chains of 10 million generations each, sampled every 1,000 generations

(BEAST v2.4.6 [75]). The cytb and LTAg gene multiple sequence alignments were used to infer

the divergence times among hosts and PyVs, respectively. The general time-reversible substitution model with gamma-distributed rate variation across sites and a proportion of invariable sites $(\mathrm{GTR}+\mathrm{G}+\mathrm{I})$ were assumed for both models. Other settings were: uncorrelated lognormal relaxed molecular clocks for host and PyV models with a Bayesian skyline population and exponential population models, respectively. These models chosen had the highest likelihood when compared to other models with the PathSampler application in BEAST v2.4.6 package. The host model was calibrated with divergence times between genera as inferred by Agnarsson [40] and the PyV model was calibrated following Buck et al. $[17,76]$.

638 genera was assessed by applying Mantel's correlation tests to the pairwise evolutionary distance

639 matrices for hosts and viral samples in both genera with $10^{4}$ repetitions. Additionally, the 640 matrices were also analysed against the geographical distance between sampling points, and 641 partial correlations between host and polyomavirus distance matrices were estimated controlling 642 for the correlation with the geographical distance. The correlation analysis was performed 643 considering three sets of data per genus: 1) considering only samples identified in China, 2) 
considering samples identified in China and samples identified in Africa [26], and 3) removing samples from 2) such as the correlation index increased. Removed samples in 3) were chosen by estimating the correlation coefficient without each sample and then removing samples whose

647 absence maximized the correlation between hosts and PyVs.

\section{Co-phylogenetic analysis.}

650 Mapping the divergence of PyV species to the divergence among hosts was performed with

651 JANE 4 [77]. The solutions for Alpha- and Betapolyomavirus genera were generated with costs

652 for co-speciation, duplication, loss and host switch events as $0,1,1$ and 3, respectively. The input

653 was the phylogenetic tree for the host species inferred with mitochondrial cytb sequences, the

654 phylogenetic trees for the Alpha- and Betapolyomavirus genera inferred with amino acid

655 sequences of the LTAg protein and the table indicating the association between hosts and viruses.

657 Analysis of natural selection on the evolution of proteins in Rhinolophus PyV species.

658 The $\omega$ ratio $(\mathrm{dN} / \mathrm{dS})$ was estimated for each coding region in clades of Rhinolophus PyV species

659 in Alpha- and Betapolyomavirus genera with MrBayes v3.2.6 [72] with NY98 as the codon 660 model for $5 \times 10^{4}$ generations sampling every 250 and assuring the effective sample size was $>$ 661100 for estimated parameters. Tridimensional structure of the VP1 protein was modeled by 662 homology with SWISS-MODEL [78]. 
664

665 1. Wilson DE, Reeder DM. Mammal species of the world: a taxonomic and geographic

666

667

668

669

670

671

672

673

674

675

676

677

678

679

680

681

682

683

684

\section{References}

reference, 3rd ed, Order Chiroptera. Baltimore, MD: Johns Hopkins University Press; 2005.

2. Streicker DG, Turmelle AS, Vonhof MJ, Kuzmin IV, McCracken GF, Rupprecht CE. Host phylogeny constrains cross-species emergence and establishment of rabies virus in bats. Science. 2010;329(5992):676. doi: 10.1126/science.1188836.

3. Towner JS, Pourrut X, Albariño CG, Nkogue CN, Bird BH, Grard G, et al. Marburg virus infection detected in a common African bat. PLoS One. 2007;2(8):e764. doi: 10.1371/journal.pone.0000764.

4. Leroy EM, Kumulungui B, Pourrut X, Rouquet P, Hassanin A, Yaba P, et al. Fruit bats as reservoirs of Ebola virus. Nature. 2005;438(7068):575. doi: 10.1038/438575a.

5. Chua KB, Lek Koh C, Hooi PS, Wee KF, Khong JH, Chua BH, et al. Isolation of Nipah virus from Malaysian Island flying-foxes. Microb Infect. 2002;4(2):145-51. doi: $10.1016 / \mathrm{S} 1286-4579(01) 01522-2$.

6. Li W, Shi Z, Yu M, Ren W, Smith C, Epstein JH, et al. Bats are natural reservoirs of SARS-like coronaviruses. Science. 2005;310(5748):676-9. doi: 10.1126/science.1118391.

7. Lau SK, Woo PC, Li KS, Huang Y, Tsoi H-W, Wong BH, et al. Severe acute respiratory syndrome coronavirus-like virus in Chinese horseshoe bats. Proc Natl Acad Sci U S A. 2005;102(39):14040-5. doi: 10.1073/pnas.0506735102.

8. He B, Zhang Y, Xu L, Yang W, Yang F, Feng Y, et al. Identification of diverse 
alphacoronaviruses and genomic characterization of a novel severe acute respiratory

688 9. Zhou P, Fan H, Lan T, Yang X-L, Shi W-F, Zhang W, et al. Fatal swine acute diarrhoea

doi: 10.1038/s41586-018-0010-9.

691 10. Wolfe ND, Dunavan CP, Diamond J. Origins of major human infectious diseases. Nature.

693 11. DeCaprio JA, Imperiale MJ, Major EO. Polyomaviridae, Fields virology. 6th ed.

695 12. Moens U, Krumbholz A, Ehlers B, Zell R, Johne R, Calvignac-Spencer S, et al. Biology,

698 13. Feng H, Shuda M, Chang Y, Moore PS. Clonal integration of a polyomavirus in human 699 Merkel cell carcinoma. Science. 2008;319(5866):1096. doi: 10.1126/science.1152586.

700 14. Dalianis T, Hirsch HH. Human polyomaviruses in disease and cancer. Virology. 701 2013;437(2):63-72. doi: 10.1016/j.virol.2012.12.015.

702 15. Dela Cruz FN, Giannitti F, Li L, Woods LW, Del Valle L, Delwart E, et al. Novel 703 polyomavirus associated with brain tumors in free-ranging raccoons, western United States. Emerg Infect Dis. 2013;19(1):77-84. doi: 10.3201/eid1901.121078.

705 16. Moens U, Calvignac-Spencer S, Lauber C, Ramqvist T, Feltkamp MCW, Daugherty MD, 
et al. ICTV virus taxonomy profile: Polyomaviridae. J Gen Virol. 2017;98(6):1159-60.

707

doi: 10.1099/jgv.0.000839.

708 17. Buck CB, Van Doorslaer K, Peretti A, Geoghegan EM, Tisza MJ, An P, et al. The ancient evolutionary history of polyomaviruses. PLoS Pathog. 2016;12(4):e1005574. doi: 10.1371/journal.ppat.1005574.

711 18. Perez-Losada M, Christensen RG, McClellan DA, Adams BJ, Viscidi RP, Demma JC, et al. Comparing phylogenetic codivergence between polyomaviruses and their hosts. J Virol. 2006;80(12):5663-9. doi: 10.1128/jvi.00056-06.

714 19. Misra V, Dumonceaux T, Dubois J, Willis C, Nadin-Davis S, Severini A, et al. Detection 10.1099/vir.0.010694-0. polyomaviruses in South American bats and their relationship to other members of the family Polyomaviridae. J Gen Virol. 2012;93(12):2652-7. doi: 10.1099/vir.0.044149-0. Virol. 2013;94(4):738-48. doi: 10.1099/vir.0.047928-0.

723 22. Lima FES, Cibulski SP, Witt AA, Franco AC, Roehe PM. Genomic characterization of 724 two novel polyomaviruses in Brazilian insectivorous bats. Arch Virol. 2015;160(7):1831-6. doi: 10.1007/s00705-015-2447-6.

726 23. Kobayashi S, Sasaki M, Nakao R, Setiyono A, Handharyani E, Orba Y, et al. Detection of 
729 24. Wang J, Moore NE, Murray ZL, McInnes K, White DJ, Tompkins DM, et al. Discovery short-tailed bat (Mystacina tuberculata). J Gen Virol. 2015;96(8):2442-52. doi: 10.1099/vir.0.000158. short-range host-switching events. J Gen Virol. 2017;98(11):2771-85. doi:

27. Johne R, Enderlein D, Nieper H, Müller H. Novel polyomavirus detected in the feces of a chimpanzee by nested broad-spectrum PCR. J Virol. 2005;79(6):3883-7. doi: 10.1128/JVI.79.6.3883-3887.2005.

743 28. Wu Z, Yang L, Ren X, He G, Zhang J, Yang J, et al. Deciphering the bat virome catalog to better understand tha ecological diversity of bat viruses and the bat origin of emerging infectious diseases. The ISME Journal. 2015;2015:1-12. doi: 10.1038/ismej.2015.138.

746 29. Cantalupo PG, Buck CB, Pipas JM. Complete genome sequence of a polyomavirus 
Genome Announc. 2017;5(3):e01053-16. doi: 10.1128/genomeA.01053-16.

749 30. Mishra N, Fagbo SF, Alagaili AN, Nitido A, Williams SH, Ng J, et al. A viral metagenomic survey identifies known and novel mammalian viruses in bats from Saudi Arabia. PLoS One. 2019;14(4):e0214227. doi: 10.1371/journal.pone.0214227.

31. Hsieh H-M, Chiang H-L, Tsai L-C, Lai S-Y, Huang N-E, Linacre A, et al. Cytochrome $b$ gene for species identification of the conservation animals. Forensic Sci Int. $2001 ; 122(1): 7-18$.

32. He B, Huang X, Zhang F, Tan W, Matthijnssens J, Qin S, et al. Group A rotaviruses in

33. $\mathrm{Xu} \mathrm{L,} \mathrm{Wu} \mathrm{J,} \mathrm{Li} \mathrm{Q,} \mathrm{Wei} \mathrm{Y,} \mathrm{Tan} \mathrm{Z,} \mathrm{Cai} \mathrm{J,} \mathrm{et} \mathrm{al.} \mathrm{Seroprevalence,} \mathrm{cross} \mathrm{antigenicity} \mathrm{and}$ analyses. PLoS Pathog. 2019;15(1):e1007545. doi: 10.1371/journal.ppat.1007545.

34. He B, Li Z, Yang F, Zheng J, Feng Y, Guo H, et al. Virome profiling of bats from 
769 37. Sun K, Feng J, Zhang Z, Xu L, Liu Y. Cryptic diversity in Chinese rhinolophids and

770 hipposiderids (Chiroptera: Rhinolophidae and Hipposideridae). Mammalia. 2009;73(2):135-41. doi: 10.1515/MAMM.2009.022.

772 38. Tan Z, Yu H, Xu L, Zhao Z, Zhang $\mathrm{P}, \mathrm{Qu} \mathrm{Y}$, et al. Virome profiling of rodents in Xinjiang Uygur Autonomous Region, China: Isolation and characterization of a new strain of Wenzhou virus. Virology. 2019;529:122-34. doi: 10.1016/j.virol.2019.01.010.

39. Dool SE, Puechmaille SJ, Foley NM, Allegrini B, Bastian A, Mutumi GL, et al. Nuclear introns outperform mitochondrial DNA in inter-specific phylogenetic reconstruction: lessons from horseshoe bats (Rhinolophidae: Chiroptera). Mol Phylogenet Evol. 2016;97:196-212. doi: 10.1016/j.ympev.2016.01.003.

40. Agnarsson I, Zambrana-Torrelio CM, Flores-Saldana NP, May-Collado LJ. A time-calibrated species-level phylogeny of bats (Chiroptera, Mammalia). PLoS currents. 2011;3:Rrn1212. doi: 10.1371/currents.RRN1212.

41. Meredith RW, Janečka JE, Gatesy J, Ryder OA, Fisher CA, Teeling EC, et al. Impacts of the Cretaceous Terrestrial Revolution and KPg extinction on mammal diversification. Science. 2011;334(6055):521. doi: 10.1126/science.1211028.

42. Stehle T, Yan Y, Benjamin TL, Harrison SC. Structure of murine polyomavirus complexed with an oligosaccharide receptor fragment. Nature. 1994;369(6476):160-3. doi: 10.1038/369160a0. 
10.1073/pnas.0710301105.

791 44. Neu U, Maginnis MS, Palma AS, Stroh LJ, Nelson CD, Feizi T, et al. Structure-function

analysis of the human JC polyomavirus establishes the LSTc pentasaccharide as a

functional receptor motif. Cell Host Microbe. 2010;8(4):309-19. doi:

45. Neu U, Hengel H, Blaum BS, Schowalter RM, Macejak D, Gilbert M, et al. Structures of

Merkel cell polyomavirus VP1 complexes define a sialic acid binding site required for infection. PLoS Pathog. 2012;8(7):e1002738. doi: 10.1371/journal.ppat.1002738.

46. Hurdiss DL, Frank M, Snowden JS, Macdonald A, Ranson NA. The structure of an infectious human polyomavirus and its interactions with cellular receptors. Structure. 2018;26(6):839-47.e3. doi: 10.1016/j.str.2018.03.019.

47. Warden CD, Lacey SF. Updated phylogenetic analysis of polyomavirus-host co-evolution.

803 48. Krumbholz A, Bininda-Emonds OR, Wutzler P, Zell R. Evolution of four BK virus subtypes. Infect Genet Evol. 2008;8(5):632-43. doi: 10.1016/j.meegid.2008.05.006.

805 49. Johne R, Muller H. Polyomaviruses of birds: etiologic agents of inflammatory diseases in a tumor virus family. J Virol. 2007;81(21):11554-9. doi: 10.1128/jvi.01178-07.

807 50. Nie FY, Lin XD, Hao ZY, Chen XN, Wang ZX, Wang MR, et al. Extensive diversity and 808 evolution of hepadnaviruses in bats in China. Virology. 2018;514:88-97. doi: $809 \quad$ 10.1016/j.virol.2017.11.005.

810 51. Eckerle I, Ehlen L, Kallies R, Wollny R, Corman VM, Cottontail VM, et al. Bat airway 
epithelial cells: a novel tool for the study of zoonotic viruses. PLoS One.

813 52. Banerjee A, Rapin N, Miller M, Griebel P, Zhou Y, Munster V, et al. Generation and

817 53. García-Pérez R, Ibáñez C, Godínez JM, Aréchiga N, Garin I, Pérez-Suárez G, et al. Novel

821 54. Corman VM, Ithete NL, Richards LR, Schoeman MC, Preiser W, Drosten C, et al.

827 56. Cook L. Polyomaviruses, Diagnostic microbiology of the immunocompromised host. 2 ed. $828 \quad$ Washington, DC: ASM Press; 2016.

829 57. Beckervordersandforth J, Pujari S, Rennspiess D, Speel EJ, Winnepenninckx V, Diaz C, 
832 58. Hausen Hz. Red meat consumption and cancer: reasons to suspect involvement of bovine infectious factors in colorectal cancer. Int J Cancer. 2012;130(11):2475-83. doi: 10.1002/ijc.27413.

59. Sauerbrei A, Wutzler P. Testing thermal resistance of viruses. Arch Virol. 2009;154(1):115-9. doi: 10.1007/s00705-008-0264-x.

60. Anthony SJ, Islam A, Johnson C, Navarrete-Macias I, Liang E, Jain K, et al. Non-random patterns in viral diversity. Nat Commun. 2015;6:8147. doi: 10.1038/ncomms9147. N-glycolyl GM1 ganglioside as a receptor for simian virus 40 . J Virol. 2007;81(23):12846-58. doi: 10.1128/jvi.01311-07.

62. O'Hara SD, Stehle T, Garcea R. Glycan receptors of the Polyomaviridae: Structure, function, and pathogenesis. Curr Opin Virol. 2014;7:73-8. doi:

845 63. Jin L, Gibson PE, Knowles WA, Clewley JP. BK virus antigenic variants: sequence analysis within the capsid VP1 epitope. J Med Virol. 1993;39(1):50-6. doi:

64. Erickson KD, Garcea RL, Tsai B. Ganglioside GT1b is a putative host cell receptor for the Merkel cell polyomavirus. J Virol. 2009;83(19):10275-9. doi: 10.1128/jvi.00949-09. 
853

854

855

856

857

858

859

860

861

862

863

864

865

866

867

868

869

870

871

872

69. Orba Y, Kobayashi S, Nakamura I, Ishii A, Hang, apos, et al. Detection and characterization of a novel polyomavirus in wild rodents. J Gen Virol. 2011;92(4):789-95. doi: doi:10.1099/vir.0.027854-0.

70. Muhire BM, Varsani A, Martin DP. SDT: A virus classification tool based on pairwise sequence alignment and identity calculation. PLoS One. 2014;9(9):e108277. doi: 10.1371/journal.pone.0108277.

71. Katoh K, Standley DM. MAFFT multiple sequence alignment software version 7: Improvements in performance and usability. Mol Biol Evol. 2013;30(4):772-80. doi: 10.1093/molbev/mst010.

873

66. Fleury MJ, Nicol JT, Samimi M, Arnold F, Cazal R, Ballaire R, et al. Identification of the neutralizing epitopes of Merkel cell polyomavirus major capsid protein within the BC and EF surface loops. PLoS One. 2015;10(3):e0121751. doi: 10.1371/journal.pone.0121751.

67. Ströh LJ, Neu U, Blaum BS, Buch MHC, Garcea RL, Stehle T. Structure analysis of the major capsid proteins of human polyomaviruses 6 and 7 reveals an obstructed sialic acid binding site. J Virol. 2014;88(18):10831. doi: 10.1128/JVI.01084-14.

68. Teeling EC, Vernes SC, Davalos LM, Ray DA, Gilbert MTP, Myers E. Bat biology, genomes, and the Bat1K project: To generate chromosome-level genomes for all Living bat species. Annu Rev Anim Biosci. 2018;6:23-46. doi: 10.1146/annurev-animal-022516-022811. 
space. Syst Biol. 2012;61(3):539-42. doi: 10.1093/sysbio/sys029.

875 73. Carr M, Kawaguchi A, Sasaki M, Gonzalez G, Ito K, Thomas Y, et al. Isolation of a simian immunodeficiency virus from a malbrouck (Chlorocebus cynosuros). Arch Virol. 2017;162(2):543-8. Epub 2016/11/03. doi: 10.1007/s00705-016-3129-8.

878 74. Kumar S, Stecher G, Tamura K. MEGA7: molecular evolutionary genetics analysis version 7.0 for bigger datasets. Mol Biol Evol. 2016;33(7):1870-4. doi: 10.1093/molbev/msw054.

881 75. Drummond AJ, Suchard MA, Xie D, Rambaut A. Bayesian phylogenetics with BEAUti and the BEAST 1.7. Mol Biol Evol. 2012;29(8):1969-73. doi: 10.1093/molbev/mss075.

883 76. Perelman P, Johnson WE, Roos C, Seuánez HN, Horvath JE, Moreira MAM, et al. A molecular phylogeny of living primates. PLoS Genet. 2011;7(3):e1001342. doi: 10.1371/journal.pgen.1001342.

886 77. Conow C, Fielder D, Ovadia Y, Libeskind-Hadas R. Jane: a new tool for the cophylogeny reconstruction problem. Algorithms Mol Biol. 2010;5(1):16. doi: 10.1186/1748-7188-5-16.

889 78. Waterhouse A, Bertoni M, Bienert S, Studer G, Tauriello G, Gumienny R, et al. 


\section{Figure legends}

Fig 1. Sampling locations and species composition of animals collected in different

provinces. The filled circles represent the locations of sampled colonies encoded by numbers

(see details in S1 File) with the red ones representing PyV-positive colonies or mammalian species and the grey ones representing the PyV-negative colonies or mammalian species (species names of PyV negative bats are not shown). The composition of collected bats or rodents in each province is presented by the colored rings with total numbers in the rings. The color region in the rings represents each positive bat families or rodent species as indicated in the top right-hand corner. Every filled circle around the colored rings represents one mammalian species. The numbers of PyV positive bats in each species are shown in parentheses. Abbreviations of provinces: XJ, Xinjiang Uyghur Autonomous Region; YN, Yunnan Province; GX, Guangxi

904 Zhuang Autonomous Region; FJ, Fujian Province; ZJ, Zhejiang Province. Abbreviations of bat genera: H., Hipposideros; A., Aselliscus; Rh., Rhinolophus; S., Scotophilus; Mi., Miniopterus; My., Myotis; T., Taphozous; Ro., Rousettus.

Fig 2. Bayesian phylogenetic tree of partial VP1 nucleotide sequences from PyV positive bats. The tree was constructed with 46 partial VP1 sequences (non-black-colored) of 44 new PyVs in present study, and $93 \mathrm{PyV}$ references (including 38 reference bat PyV species) in Alpha911 and Betapolyomavirus genera available in GenBank. The names of putative novel PyV strains are 912 differentiated using colors according to the Chiropteran family of bat hosts following the legend 913 in the top right-hand corner. The PyV positive rate of each putative PyV species from different 914 colonies is shown in red in parentheses next to the tip names. Each parenthesis represents one 915 colony. Host-switching events are marked in the clusters showing the presence of closely related 
916 PyVs with different bat host species (HS1-HS4). The posterior probability supporting the

917 branching is shown next to the branch. Scale: 0.2 nucleotide mutations per site.

Fig 3. Pairwise identity comparison of the LTAg genes of bat-borne PyVs identified in the PyV) by circles.

Fig 4. Bayesian phylogenetic tree of the PyV LTAg protein sequences. The tree was constructed with 156 amino acid sequences of 28 new PyVs identified in the present study, and $126 \mathrm{PyV}$ references (including 38 bat PyV species references) in Alpha- and Betapolyomavirus genera available in GenBank. Names of new PyVs with their strain names in parentheses at the branch tips are colored differently according to their host species within the Chiropteran family, as shown in the top left-hand corner. Three host-switching events are marked as HS1 to HS3 by

934 opposing arrows. Host switching lineages identified by correlation analyses are also shown with 935 black circles. Silhouettes of the mammalian hosts of PyVs are shown alongside each clade. Bayesian posterior probability supporting the branching is shown next to the branches.

937 Abbreviations of PyVs: Ra-Hl PyV, Rhinolophus affinis-Hipposideros larvatus polyomavirus;

938 Ra-Mh PyV, Rhinolophus affinis-Myotis horsfieldii polyomavirus. 
Fig 5. Dating the tMRCA of host bat species employing mitochondrial cytb sequences (A) and PyV species using the LTAg gene (B). Cytb and PyV sequences from this study are colored according to the Chiropteran family of the host species and following the legend in the top

942 right-hand corner. Calibration points of $c y t b$ are marked on the branches with black squares (A).

943 Calibration points using PyVs identified from non-human primates and other bat species are

944 shown in the upper section of the phylogeny and marked with black squares (B). The timescale at 945 the bottom of both figures and the tMRCAs shown next to the branches are in millions of years 946 (MYA) before the present. Median age of the tMRCAs of cytb (A) and PyVs (B) are shown next 947 to the branches. In Fig 5B, branches that matched the divergence between host species in Fig 5A 948 are identified with red circles.

951 their hosts. The blue phylogenies show the inferred host divergence. The red phylogenies are 952 annotated to reconstruct the divergence among viral species according to JANE 4 in the 
963 the host species, (C) viral duplication in the same host species and (D) duplication and

964 host-switching to a diverged (susceptible) host species. The phylogenies close to the cartoons are

965 the expected phylogenetic relationship for each respective case with the events marked at the

966 nodes.

968 Supporting information

970 PyV positive samples. The tree was constructed with 192 partial VP1 sequences of PyVs in

971 present study (filled circles), and $74 \mathrm{PyV}$ references (including 38 bat PyV species references) in

972 Alpha- and Betapolyomavirus genera downloaded from GenBank. These 192 partial VP1

973 sequences were classified into 44 clusters representing host-switching events (green circles;

974 clusters 7, 10, 17 and 31; red circles: no host switching). GenBank accession numbers of 192

975 partial PyV VP1 sequences in this tree are listed in S3 Table. The tree was generated in MEGA7

976 by the maximum-likelihood method based on the TN93 model and evaluated with 250 bootstrap

977 replicates.

978

979 S2 Fig. Bayesian phylogenetic tree of the cytb sequences of bats. The tree was constructed 980 with 37 reference bat $c y t b$ genes and 42 bat $c y t b$ genes in the present study (colored differently 981 according to the bat families) for confirmation of the species of bats from which PyV full 982 genomes were obtained. The posterior probability supporting the branching is shown next to the 983 branch. GenBank accession numbers of $c y t b$ sequences in this tree are listed in S4 Table. 
Alpha- and Betapolyomavirus genera. The top panels show the $\omega$ rate $(\mathrm{dN} / \mathrm{dS})$ estimated with a

987

988

989

990

991

992

993

994

995

996

997

998

999

1000

1001

1002

1003

1004

1005

1006

1007
Bayesian approach for clusters of Rhinolophus PyVs in both Alpha- and Betapolyomavirus genera with positions adjusted to Rhinolophus affinis polyomavirus 1 [PyVB-FN04C] and Rhinolophus affinis polyomavirus 3 [PyV19-SWAC9], respectively. Tridimensional models for the VP1 proteins corresponding to both genera were inferred by structure homology modelling. The top and bottom views of both modelled proteins are shown in gray with sites under positive selection colored in red.

S1 Table. Summary of sampled colonies and detailed information of PyV positive bats following a geographic approach of the collection sites.

S2 Table. Summary of detected PyVs and their positive rates in colonies.

S3 Table. GenBank accession numbers of partial PyV VP1 sequences.

S4 Table. GenBank accession numbers of $1.8 \mathrm{~kb}$ cytb sequences.

S5 Table. VP1 amino acid residues under positive selection in Rhinolophus affinis polyomavirus 1 in the Alphapolyomavirus genus and Rhinolophus affinis polyomavirus 3 in the Betapolyomavirus genus with homologous positions relative to human BK virus (species of Human polyomavirus 1) and Simian virus 40 (species of Macaca mulatta polyomavirus 1). 

aCC-BY 4.0 International license.

1009 S6 Table. List of oligonucleotides used in the present study. 


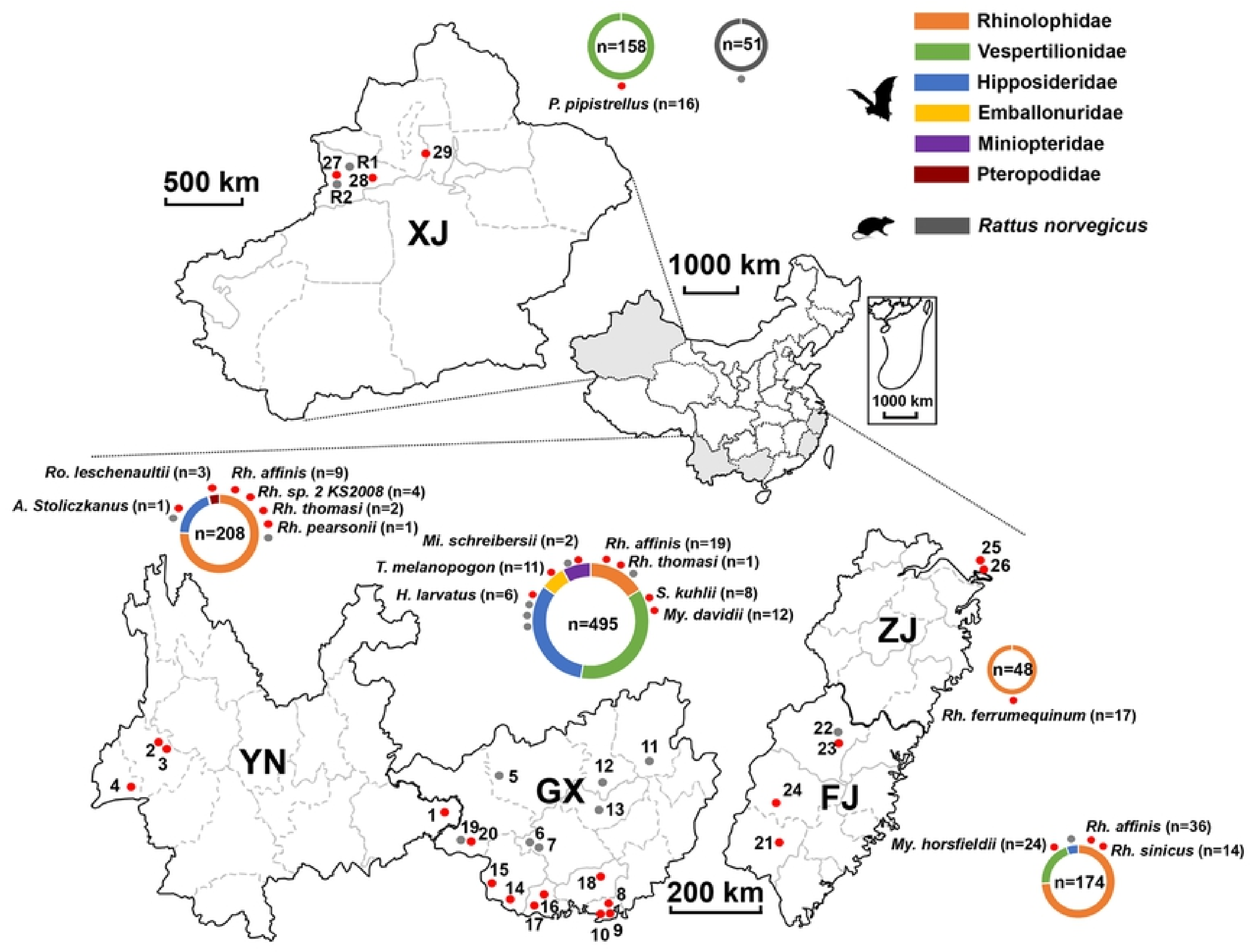

Figure 1 


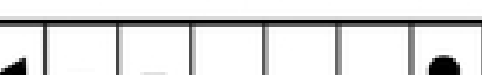

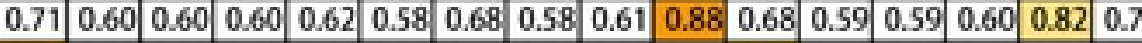

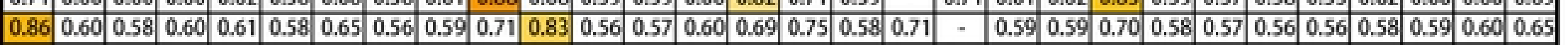

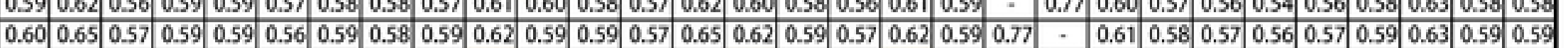

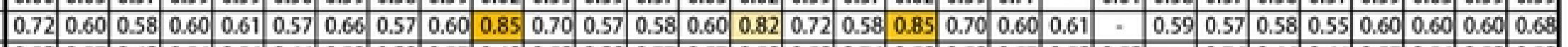

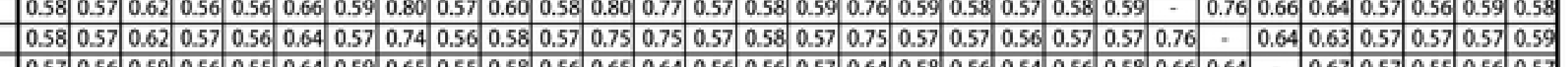

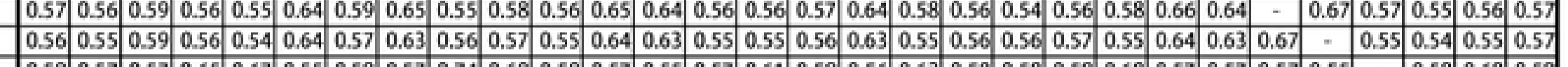

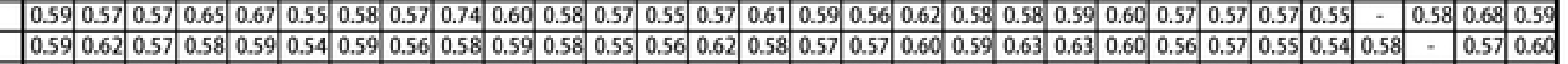

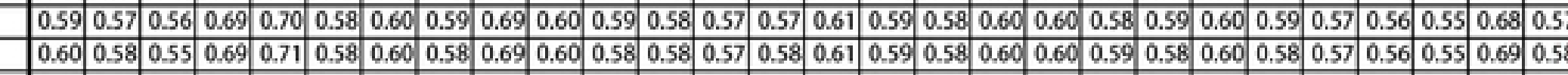

\begin{tabular}{l} 
Rhinologhrus simulator polyomavirus I (LC269978) \\
\hline Carollig perspicilloto polyomavirus I (J0958889) \\
\hline
\end{tabular}

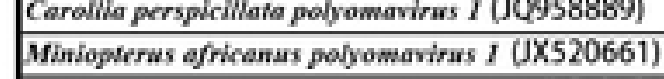
Miniopterus schreibersiij polyomavirus I (LC185215) Molossus molossus potyomavirus 1 (JQ958893)

Myofis pequinius polyomavirus I (KJ641707)

Rhinolophus simulator polyomavirus 3 (LC269980) Rhinolophus simulator polyomavirus 2 (LC269979)

Chaerephon polyomanirus I (NC_020065)

Rhinelophus Blasii pelyomavirus 2(LC269977)

\begin{tabular}{|l|}
\hline Cardioderma cor polyomavirus I (JX520659) \\
\hline Otamops martiensseni polyomavirus 2 (JX52066: \\
\hline
\end{tabular}

Acerodon celebensis polyomavirus 2 (AB972941)

Dobsomia moluccensis polyomavirus 3 (AB972946) 



Figure 4 

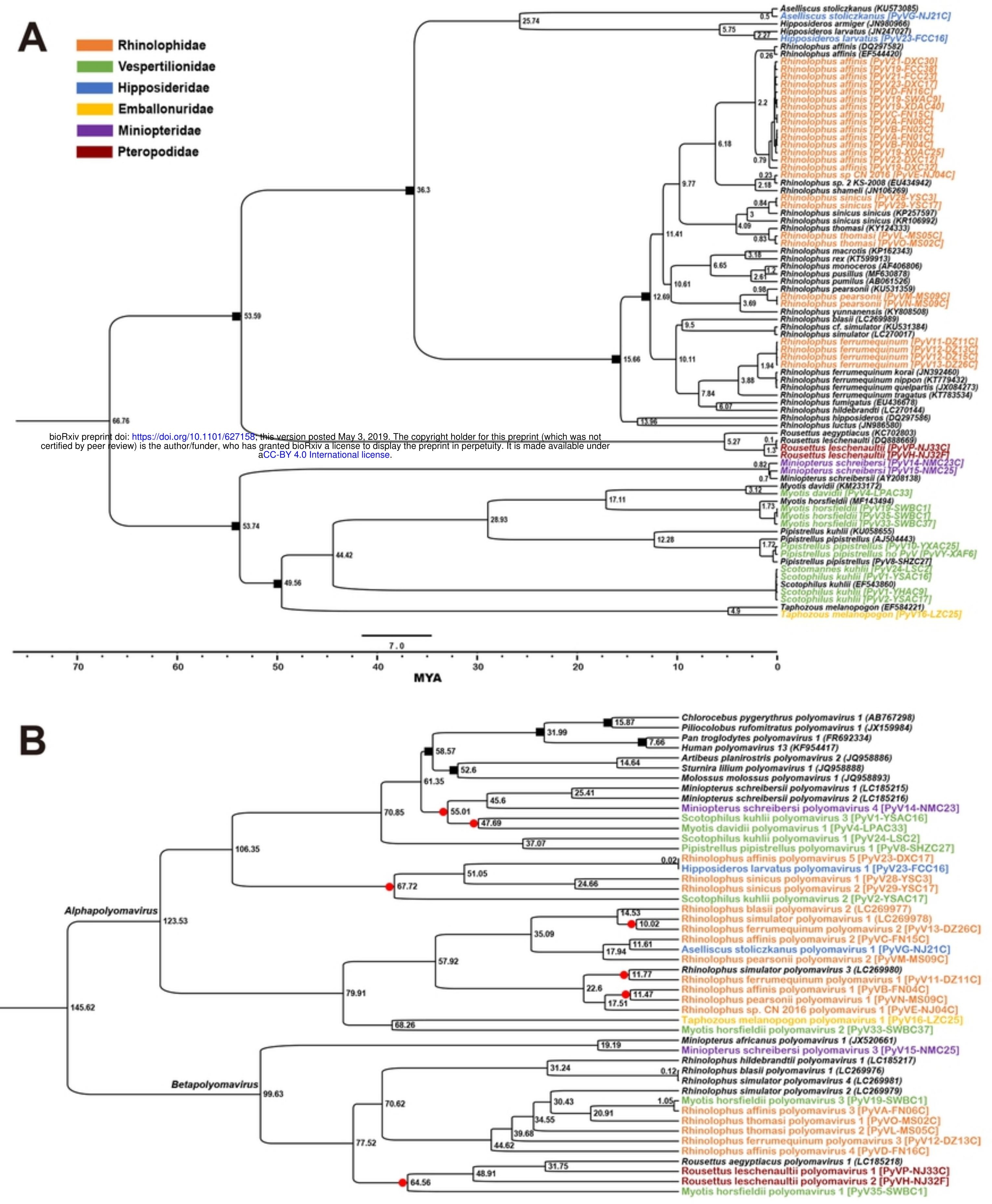

\begin{tabular}{|c|c|c|c|c|c|}
\hline & & & 20.0 & & \\
\hline${ }_{150}^{1}$ & $\begin{array}{c}1 \\
125\end{array}$ & ${ }_{100}^{1}$ & $\begin{array}{l}1 \\
75\end{array}$ & so & $\begin{array}{l}1 \\
25\end{array}$ \\
\hline & & & MYA & & \\
\hline
\end{tabular}

\section{Figure 5}


A

B

Virus phylogeny

Virus phylogeny

A

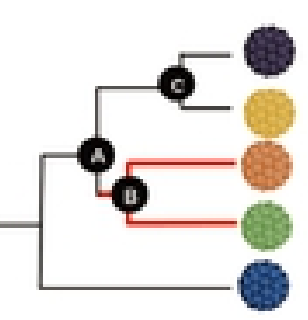

B

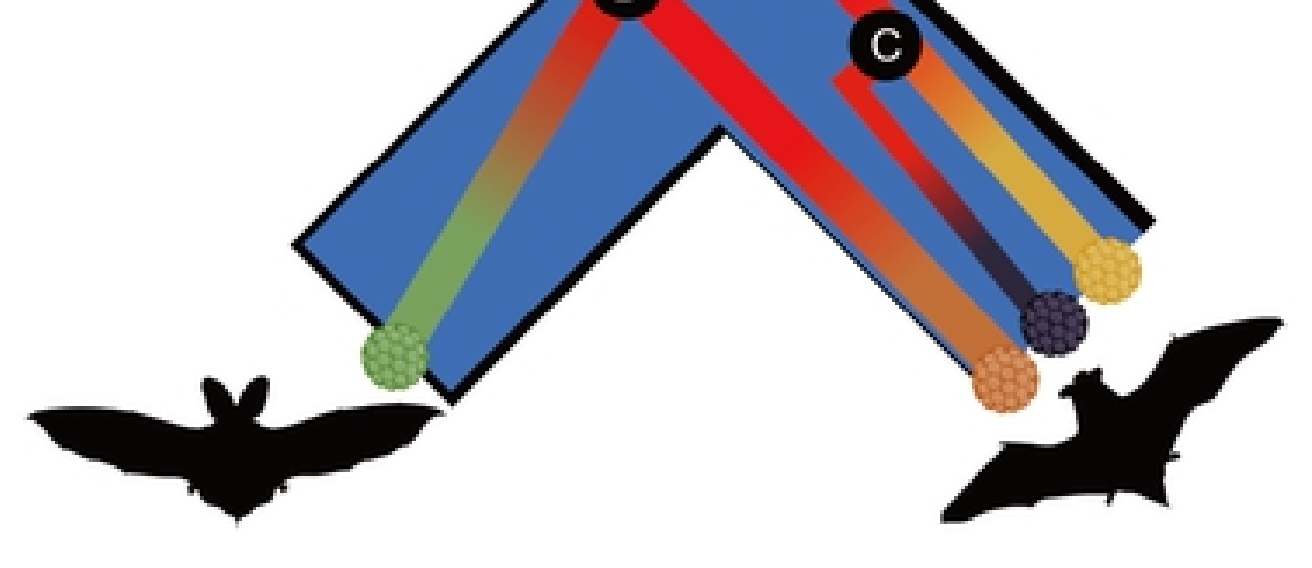

C

Figure 7 


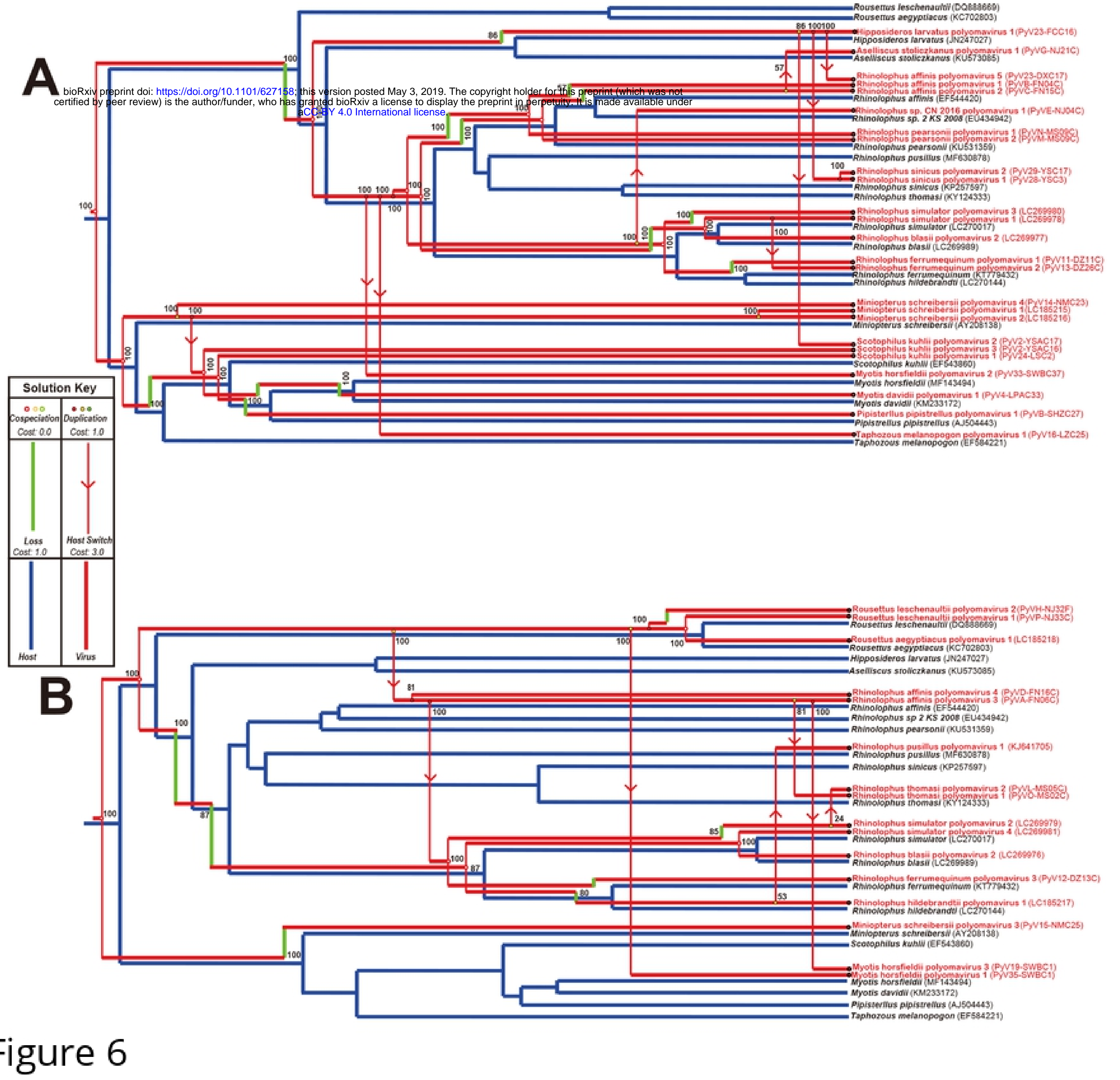

